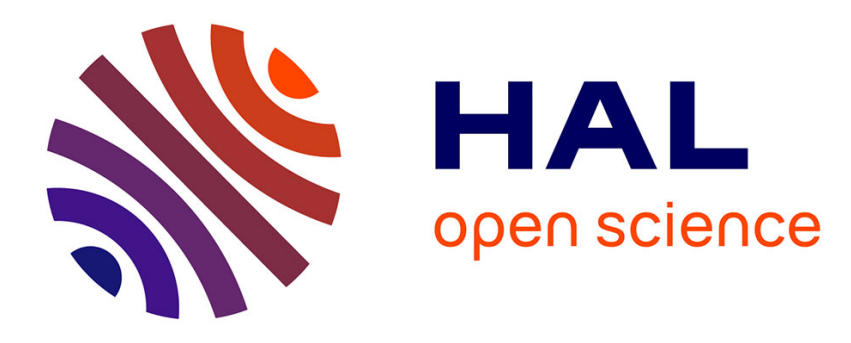

\title{
Time-integration schemes for the finite element dynamic Signorini problem
}

\author{
David Doyen, Alexandre Ern, Serge Piperno
}

\section{To cite this version:}

David Doyen, Alexandre Ern, Serge Piperno. Time-integration schemes for the finite element dynamic Signorini problem. SIAM Journal on Scientific Computing, 2011, 33 (1), pp.223-249. 10.1137/100791440 . hal-00440128

\section{HAL Id: hal-00440128 \\ https://hal.science/hal-00440128}

Submitted on 9 Dec 2009

HAL is a multi-disciplinary open access archive for the deposit and dissemination of scientific research documents, whether they are published or not. The documents may come from teaching and research institutions in France or abroad, or from public or private research centers.
L'archive ouverte pluridisciplinaire HAL, est destinée au dépôt et à la diffusion de documents scientifiques de niveau recherche, publiés ou non, émanant des établissements d'enseignement et de recherche français ou étrangers, des laboratoires publics ou privés. 


\title{
Time-integration schemes for the finite element dynamic Signorini problem
}

\author{
D. Doyen ${ }^{1,2, *}$, A. Ern ${ }^{2}$ and S. Piperno ${ }^{2}$ \\ 1 EDF RछD, 1 avenue du Général de Gaulle, 92141 Clamart Cedex, France \\ 2 Université Paris-Est, CERMICS, Ecole des Ponts, 77455 Marne la Vallée Cedex 2, France
}

\begin{abstract}
SUMMARY
The discretization of the dynamic Signorini problem with finite elements in space and a time-stepping scheme is not straightforward. Consequently a large variety of methods for this problem have been designed over the last two decades. Up to date, no systematic comparison of such methods has been performed. The aim of the present work is to classify and compare them. For each method, we discuss the presence of spurious oscillations and the energy conservation. For explicit approaches, the stability condition on the time step is also discussed. Numerical simulations on two 1D benchmark problems with analytical solutions illustrate the properties of the different methods. Most of the discretizations considered herein can be found in the literature, but the semi-explicit modified mass method is new and features, in our opinion, several attractive properties. Copyright (c) 2000 John Wiley \& Sons, Ltd.

KEY WORDS: elastodynamics; frictionless unilateral contact; time-integration schemes; finite elements; modified mass method
\end{abstract}

\section{INTRODUCTION}

The design of robust and efficient numerical methods for dynamic contact problems has motivated a large amount of work over the last two decades and remains a challenging issue. Here, we focus on the dynamic Signorini problem, which models the infinitesimal deformations of a solid body that can come into contact with a rigid obstacle. This problem is the simplest dynamic contact problem, but also the first step toward more complex situations, such as multi-body problems, large deformation problems, contact with friction, etc... For an overview of the different contact problems, we refer to [19, 21, 31].

In structural dynamics, the usual space-time discretization combines finite elements in space and a time-stepping scheme. In this framework, the discretization of the dynamic Signorini

* Correspondence to: Université Paris-Est, CERMICS, Ecole des Ponts, 77455 Marne la Vallée Cedex 2, France. ${ }^{\dagger}$ Please ensure that you use the most up to date class file, available from the NME Home Page at http://www.interscience.wiley.com/jpages/0029-5981/

Contract/grant sponsor: Publishing Arts Research Council; contract/grant number: 98-1846389 
problem involves mainly three choices: (i) the finite element space; (ii) the enforcement of the contact condition; (iii) the time-stepping scheme. The combination of these three ingredients is not straightforward. For instance, it is well-known that the combination of an exact enforcement of the contact condition and an implicit Newmark scheme yields spurious oscillations as well as poor energy conservation and long-time behavior. Moreover, the combination of an exact enforcement and an explicit scheme is not straightforward, whereas the use of a penalty contact condition tightens the stability condition of explicit schemes. Consequently, various alternative discretizations have been designed for the dynamic Signorini problem. However, up to date, no systematic comparison of such methods has been performed. The aim of the present work is to classify and compare the main discretizations for the dynamic Signorini problem. Most of the discretizations considered herein can be found in the literature. The semi-explicit modified mass method (Discretization 7.2 below) is new and features, in our opinion, several attractive properties.

We classify the different discretizations into four groups. The first three groups correspond to different ways of enforcing the contact condition: exact enforcement $[5,7,17,23,25,26,29,30]$, enforcement with penalty $[1,3,14]$ and enforcement with contact condition in velocity $[2,3,22]$. The fourth approach is based on a modification of the mass matrix $[13,18]$; it can be seen as an alternative choice of the finite element space. These four classes yield different semidiscrete problems in space which in turn can be discretized in time using various time-stepping schemes, either implicit or (semi-)explicit. In our opinion, a reliable discretization should not trigger spurious oscillations during the contact phases and achieve a tight energy conservation. Energy conservation is important to guarantee a good behavior in long time. By energy conservation we mean that the variation of the energy is equal to the work of the external forces (the contact forces should not work). For each scheme, the presence of spurious oscillations and the energy conservation are examined. In addition, for explicit approaches, the stability condition on the time step is also discussed. Furthermore dynamic contact problems yield shock waves, and spurious oscillations appear near the shock in the numerical solutions, owing to the so-called Gibbs phenomenon. This issue being important but not specific to dynamic contact problems, it will be briefly addressed in the appendix. To illustrate the behavior of the different discretizations, numerical simulations on two 1D benchmark problems with analytical solutions have been performed. The first benchmark, the impact of an elastic bar, is well-known in the literature and allows to detect spurious oscillations. The second one, the bounces of an elastic bar, is geared towards the energy conservation and long-time behavior of the discretizations. It is new to our knowledge. Although both benchmarks are 1D, we believe they are representative of the various difficulties encountered in higher space dimension. Furthermore, the mathematical analysis of the different methods is beyond the scope of this article, but we mention, whenever they exist, the theoretical results (well-posedness of the discrete problems and convergence of the discrete solutions).

The article is organized as follows. We formulate the dynamic Signorini problem in the continuous setting (Section 2.1) and we introduce the main ingredients for its approximation (Sections 2.2 and 2.3). We present the two benchmark problems with their analytical solutions (Section 3). We describe the four classes of discretizations together with numerical results: exact enforcement of the contact condition (Section 4), enforcement with penalty contact condition (Section 5), enforcement with contact condition in velocity (Section 6) and modification of the mass matrix (Section 7). The Appendix is devoted to the Gibbs phenomenon. 


\section{THE DYNAMIC SIGNORINI PROBLEM}

\subsection{Governing equations}

We consider the infinitesimal deformations of a body occupying a reference domain $\Omega \subset \mathbb{R}^{d}$ $(d=1, d=2$ or $d=3)$ during a time interval $[0, T]$. The tensor of elasticity is denoted by $\mathcal{A}$ and the mass density is denoted by $\rho$. An external load $f$ is applied to the body. Let $u:(0, T) \times \Omega \rightarrow \mathbb{R}^{d}, \epsilon(u):(0, T) \times \Omega \rightarrow \mathbb{R}^{d, d}$ and $\sigma(u):(0, T) \times \Omega \rightarrow \mathbb{R}^{d, d}$ be the displacement field, the linearized strain tensor and the stress tensor, respectively. Denoting time-derivatives by dots, the equilibrium equation is

$$
\rho \ddot{u}-\operatorname{div} \sigma=f, \quad \sigma=\mathcal{A}: \epsilon, \quad \epsilon=\frac{1}{2}\left(\nabla u+{ }^{\mathrm{T}} \nabla u\right) \quad \text { in } \Omega \times(0, T) .
$$

The boundary $\partial \Omega$ is partitioned into three disjoint open subsets $\Gamma^{D}, \Gamma^{N}$ and $\Gamma^{c}$. Dirichlet and Neumann conditions are prescribed on $\Gamma^{D}$ and $\Gamma^{N}$, respectively,

$$
u=u_{D} \quad \text { on } \Gamma^{D} \times(0, T), \quad \sigma \cdot n=f_{N} \quad \text { on } \Gamma^{N} \times(0, T),
$$

where $n$ denote the outward unit normal to $\Omega$. We set $u_{n}:=u_{\mid \partial \Omega} \cdot n$ and $\sigma_{n}:=n \cdot \sigma_{\mid \partial \Omega} \cdot n$, the normal displacement and the normal stress on $\partial \Omega$, respectively. On $\Gamma^{c}$, a unilateral contact condition, also called Signorini condition, is imposed,

$$
u_{n} \leq 0, \sigma_{n}(u) \leq 0, \sigma_{n}(u) u_{n}=0 \quad \text { on } \Gamma^{c} \times(0, T) .
$$

At the initial time, the displacement and velocity fields are prescribed,

$$
u(0)=u^{0}, \quad \dot{u}(0)=v^{0} \quad \text { in } \Omega .
$$

Problem (1)-(4) is an evolution partial differential equation under unilateral constraints. Here, the equation is second-order in time and the constraint holds on the displacement; this is not the most favorable case. The existence and uniqueness of a solution has only been proven in $1 \mathrm{D}$, when the contact boundary is reduced to a point $[24,10]$. In $1 \mathrm{D}$, it has also been proven that the variation of energy is equal to the work of the external forces; the contact force does not work $[24,10]$. In higher dimension, the existence of a solution is proven in the case of a viscoelastic material [10].

\subsection{Basic time-integration schemes in linear elastodynamics}

In this section, we briefly recall some basic facts about time-integration schemes in linear elastodynamics; most of this material can be found in [15]. Firstly, we discretize the problem in space with a finite element method. The number of degrees of freedom is denoted by $N_{d}$. Let $K, M$, and $F(t)$ be the stiffness matrix, the mass matrix, and the column vector of the external forces, respectively. The space semi-discrete problem consists in seeking $u:[0, T] \rightarrow \mathbb{R}^{N_{d}}$ such that, for all $t \in[0, T]$,

$$
M \ddot{u}(t)+K u(t)=F(t),
$$

with the initial conditions $u(0)=u^{0}$ and $\dot{u}(0)=v^{0}$. For solving such a system of ODEs (ordinary differential equations), linear one-step schemes are the most frequently used. The interval $[0, T]$ is divided into equal subintervals of length $\Delta t$. We set $t^{n}=n \Delta t$ and denote by $u^{n}, \dot{u}^{n}$, and $\ddot{u}^{n}$ the approximations of $u\left(t^{n}\right), \dot{u}\left(t^{n}\right)$, and $\ddot{u}\left(t^{n}\right)$, respectively. We define the convex 
combination $\square^{n+\alpha}:=(1-\alpha) \square^{n}+\alpha \square^{n+1}$, where $\square$ stands for $u, \dot{u}, \ddot{u}$ or $t$, and $\alpha \in[0,1]$. We also set $F^{n+\alpha}:=F\left(t^{n+\alpha}\right)$. At time $t^{n}$, the energy of the system is $\mathcal{E}^{n}:=\frac{1}{2}{ }^{\mathrm{T}} \dot{u}^{n} M \dot{u}^{n}+\frac{1}{2}^{\mathrm{T}} u^{n} K u^{n}$. It is also convenient to define the quadratic form $\mathcal{E}_{\beta, \gamma}^{n}:=\mathcal{E}^{n}+\frac{\Delta t^{2}}{2}\left(\beta-\frac{1}{2} \gamma\right)^{\mathrm{T}} \ddot{u}^{n} M \ddot{u}^{n}$. Now we can formulate some of the most common time-stepping schemes in linear elastodynamics.

Discretization 2.1 (HHT-Newmark) Seek $u^{n+1}, \dot{u}^{n+1}, \ddot{u}^{n+1} \in \mathbb{R}^{N_{d}}$ such that

$$
\begin{aligned}
& M \ddot{u}^{n+1}+K u^{n+1+\alpha}=F^{n+1+\alpha}, \\
& u^{n+1}=u^{n}+\Delta t \dot{u}^{n}+\frac{\Delta t^{2}}{2} \ddot{u}^{n+2 \beta}, \\
& \dot{u}^{n+1}=\dot{u}^{n}+\Delta t \ddot{u}^{n+\gamma},
\end{aligned}
$$

where $\alpha, \beta, \gamma$ are parameters.

Discretization 2.2 (Midpoint) Seek $u^{n+1}, \dot{u}^{n+1}, \ddot{u}^{n+\frac{1}{2}} \in \mathbb{R}^{N_{d}}$ such that

$$
\begin{aligned}
& M \ddot{u}^{n+\frac{1}{2}}+K u^{n+\frac{1}{2}}=F^{n+\frac{1}{2}}, \\
& u^{n+1}=u^{n}+\Delta t \dot{u}^{n+\frac{1}{2}}, \\
& \dot{u}^{n+1}=\dot{u}^{n}+\Delta t \ddot{u}^{n+\frac{1}{2}} .
\end{aligned}
$$

Discretization 2.3 (Central differences) Seek $u^{n+1} \in \mathbb{R}^{N_{d}}$ such that

$$
M\left(\frac{u^{n+1}-2 u^{n}+u^{n-1}}{\Delta t^{2}}\right)+K u^{n}=F^{n} .
$$

When $\alpha=0$, the schemes (6)-(8) are called Newmark schemes. For these schemes, the energy balance reads [20],

$$
\begin{aligned}
& \mathcal{E}_{\beta, \gamma}^{n+1}-\mathcal{E}_{\beta, \gamma}^{n}={ }^{\mathrm{T}}\left(\frac{1}{2}\left(F^{n+1}+F^{n}\right)+\left(\gamma-\frac{1}{2}\right)\left(F^{n+1}-F^{n}\right)\right)\left(u^{n+1}-u^{n}\right) \\
& -\left(\gamma-\frac{1}{2}\right)\left({ }^{\mathrm{T}}\left(u^{n+1}-u^{n}\right) K\left(u^{n+1}-u^{n}\right)+\left(\beta-\frac{1}{2} \gamma\right){ }^{\mathrm{T}}\left(\ddot{u}^{n+1}-\ddot{u}^{n}\right) M\left(\ddot{u}^{n+1}-\ddot{u}^{n}\right)\right) .
\end{aligned}
$$

The particular choice $\beta=1 / 4, \gamma=1 / 2$ yields an implicit, unconditionally stable, and secondorder scheme. It is energy-conserving in the sense that

$$
\mathcal{E}^{n+1}-\mathcal{E}^{n}={ }^{\mathrm{T}} F^{n+1}\left(u^{n+1}-u^{n}\right) .
$$

When $\alpha \in[-1 / 3,0], \beta=1 / 4(1-\alpha)^{2}, \gamma=1 / 2-\alpha$, the schemes (6)-(8) are called HHT schemes or $\alpha$-methods. Such schemes are implicit, unconditionally stable, second-order and dissipative in the high frequencies. The amount of dissipation is controlled by the parameter $\alpha$. The midpoint scheme is implicit, unconditionally stable, and second-order. It is energy-conserving, in the sense that

$$
\mathcal{E}^{n+1}-\mathcal{E}^{n}={ }^{\mathrm{T}} F^{n+\frac{1}{2}}\left(u^{n+1}-u^{n}\right) .
$$

The central difference scheme is explicit (provided the mass matrix is lumped), conditionally stable and second-order. Here it is written as a two-step linear scheme involving only the displacement but it can be formulated as a one-step scheme. Actually, it is a Newmark scheme with parameters $\beta=0, \gamma=1 / 2$; the associated velocity and acceleration are then 
$\dot{u}^{n}=\frac{1}{2 \Delta t}\left(u^{n+1}-u^{n-1}\right)$ and $\ddot{u}^{n}=\frac{1}{\Delta t^{2}}\left(u^{n+1}-2 u^{n}+u^{n-1}\right)$. The central difference scheme conserves a quadratic form which is nearly the energy,

$$
\mathcal{E}_{0, \frac{1}{2}}^{n+1}-\mathcal{E}_{0, \frac{1}{2}}^{n}={ }^{\mathrm{T}}\left(\frac{F^{n+1}+F^{n}}{2}\right)\left(u^{n+1}-u^{n}\right) .
$$

There exist also second-order explicit schemes with high-frequency dissipation, such as the Chung-Hulbert schemes [16].

\subsection{Enforcing the contact condition}

The enforcement of a contact condition in a finite element setting has been widely studied in the case of the static Signorini problem [19]. We assume that the mesh is compatible with the partition of the boundary. Let $N_{c}$ be the number of nodes lying on the contact boundary. We define the linear normal trace operator on $\Gamma^{c}, g: v \longmapsto-v_{\mid \Gamma} \cdot n$ and the associated matrix $G$. Note that the dimension of $G$ is $N_{c} \times N_{d}$. We denote by $\left\{G_{i}\right\}_{1 \leq i \leq N_{c}}$ the rows of the matrix $G$. Thus, $G_{i} u$ yields the value of the normal displacement at the $i$ th node of the contact boundary. With an exact enforcement, the static Signorini problem consists in seeking a displacement $u \in \mathbb{R}^{N_{d}}$ and a contact pressure $r \in \mathbb{R}^{N_{c}}$ such that

$$
\begin{aligned}
& K u=F+{ }^{\mathrm{T}} G r, \\
& G u \geq 0, \quad r \geq 0, \quad{ }^{\mathrm{T}} r G u=0 .
\end{aligned}
$$

Here the problem is formulated as a complementarity problem. Other formalisms can be found in the literature, e.g., variational inequality, Lagrangian formulation and formulation with subderivatives. If the matrix $K$ is positive definite, problem (17)-(18) has a unique solution. For solving this problem, a large variety of methods has been developed [19, 31]: Uzawa algorithms, active set methods, semi-smooth Newton methods, Lemke algorithm, etc...

Penalty formulations are another classical way of dealing with constrained problems. We have to define a penalty function $R_{\epsilon}: \mathbb{R}^{N_{c}} \rightarrow \mathbb{R}^{N_{c}}$. For instance we can choose $R_{\epsilon}(v)=\frac{1}{\epsilon}(v)^{-}$, where $(v)^{-}$denotes the negative part of $v$. The penalized static Signorini problem consists now in seeking $u \in \mathbb{R}^{N_{d}}$ such that

$$
K u=F+{ }^{\mathrm{T}} G R_{\epsilon}(G u) .
$$

A third way of enforcing the contact condition, specific to the dynamic problem, is to replace the Signorini condition by an approximation involving the velocity instead of the displacement [10]. Assume that $u_{n}=0$ at a certain time $t^{c}$. Then, on a short time interval afterwards, $u_{n} \approx\left(t-t^{c}\right) \dot{u}_{n}$. This motivates the following contact condition in velocity,

$$
\dot{u}_{n} \leq 0, \sigma_{n}(u) \leq 0, \sigma_{n}(u) \dot{u}_{n}=0, \quad \text { on } \Gamma^{c} .
$$

It must be stated that this condition is not rigorously equivalent to the Signorini condition.

\section{BENCHMARK PROBLEMS}

To compare the different methods, we test them on two 1D problems. Both problems can be formulated in the same setting. We consider an elastic bar dropped against a rigid ground. The 
bar is dropped, undeformed, from a height $h_{0}$, with an initial velocity $-v_{0}$, under a gravity $g_{0} \geq 0$. The length of the bar is denoted by $L$, the Young modulus by $E$ and the density by $\rho$. Let $c_{0}:=\sqrt{\frac{E}{\rho}}$ denote the wave speed. The reference domain is $\Omega=[0, L]$. The displacement $u$ of the bar and the contact pressure $r$ satisfy the following equations,

$$
\begin{aligned}
& \rho \ddot{u}-E \frac{\partial^{2} u}{\partial x^{2}}=-\rho g_{0}+r, \quad \text { in } \Omega \times(0, T), \\
& u(0, t) \geq 0, r(t) \geq 0, r(t) u(0, t)=0 \quad \text { on }(0, T), \\
& \frac{\partial u}{\partial x}(L, t)=0 \quad \text { on }(0, T), \\
& u(\cdot, 0)=h_{0}, \quad \dot{u}(\cdot, 0)=-v_{0} .
\end{aligned}
$$

Problem (21)-(24) has a unique solution and the variation of the energy is equal to the work of the gravity force [24],

$$
\frac{d}{d t}\left(\frac{1}{2} \int_{\Omega} \rho \dot{u}^{2}+\frac{1}{2} \int_{\Omega} E\left|\frac{\partial u}{\partial x}\right|^{2}\right)=\int_{\Omega}-g_{0} \dot{u}, \quad \forall t \in[0, T] .
$$

In the first problem, $v_{0}>0$ and $g_{0}=0$. This benchmark has been widely used in the literature. It enables us to examine the possible spurious oscillations triggered by the numerical schemes. In the second problem, $v_{0}=0$ and $g_{0}>0$, so that the bar can make several bounces. With a suitable choice of parameters, the motion of the bar is periodic in time and we can calculate the exact solution. It enables us to examine the energy conservation and the long-time behavior of the different numerical methods. This benchmark is, to our knowledge, new.

\subsection{Impact of an elastic bar}

Let us describe the solution of the benchmark problem (Figure 1). Before the impact, the bar is undeformed and has a uniform velocity $-v_{0}$. The bar reaches the ground at time $t_{1}=\frac{h_{0}}{v_{0}}$. After the impact, the bar stays in contact with the ground. A shock wave travels from the bottom of the bar to the top. Above the shock wave, the velocity is $-v_{0}$; below, the velocity is zero. Then, the shock wave travels from the top to the bottom. Above the shock wave, the velocity is $v_{0}$; below, the velocity is still zero. As soon as the wave reaches the bottom, the bar takes off, undeformed, with a uniform velocity $v_{0}$. The speed of the shock wave is $c_{0}$. Thus, the wave takes a time $\tau_{w}:=\frac{L}{c_{0}}$ to cross the bar, and the bar takes off at time $t_{2}=t_{1}+2 \tau_{w}$. The analytical solution can be easily expressed using travelling functions. Defining the auxiliary function $H_{v}(x, t)=-v \min \left(x / c_{0}, \tau_{w}-\left|t-\tau_{w}\right|\right)$, the exact solution is

$$
u(x, t)= \begin{cases}h_{0}-v_{0} t & \text { if } t \leq t_{1} \\ H_{v_{0}}\left(x, t-t_{1}\right) & \text { if } t_{1}<t \leq t_{2} \\ v_{0}\left(t-t_{2}\right) & \text { if } t_{2}<t\end{cases}
$$

In particular the displacement at the bottom of the bar and the contact pressure are:

$$
u(0, t)=\left\{\begin{array}{ll}
h_{0}-v_{0} t & \text { if } t \leq t_{1} \\
0 & \text { if } t_{1}<t \leq t_{2} \\
v_{0}\left(t-t_{2}\right) & \text { if } t_{2}<t
\end{array} \quad r(t)= \begin{cases}0 & \text { if } t \leq t_{1} \\
\frac{E v_{0}}{c_{0}} & \text { if } t_{1}<t \leq t_{2} \\
0 & \text { if } t_{2}<t\end{cases}\right.
$$

These two quantities are represented in Figure 2 (with the parameters chosen in Section 3.3). 


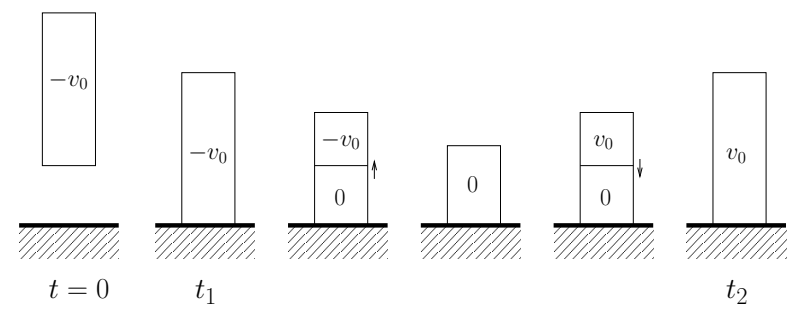

Figure 1. Impact of an elastic bar.
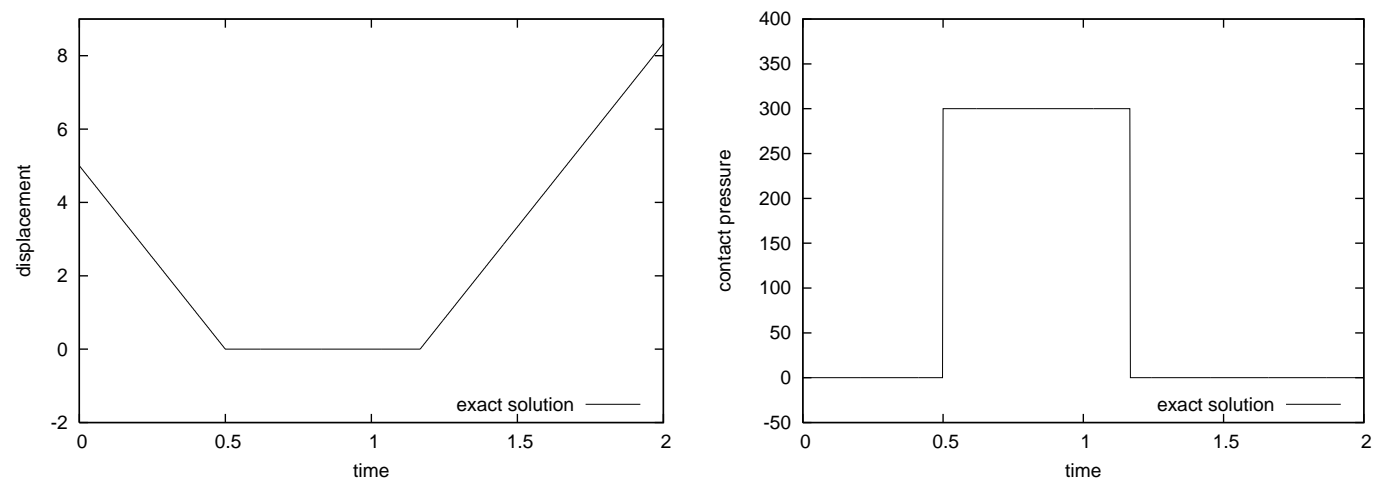

Figure 2. Impact of an elastic bar. Displacement at the bottom of the bar (left) and contact pressure (right).

\subsection{Bounces of an elastic bar}

In this benchmark problem (Figure 3), the bar is dropped, undeformed, with a zero velocity. It takes a time $\tau_{f}:=\sqrt{\frac{2 h_{0}}{g_{0}}}$ to reach the ground. At the impact, at time $t_{1}:=\tau_{f}$, the bar is undeformed and has uniform velocity $-v_{f}$, where $v_{f}:=\sqrt{2 h_{0} g_{0}}$. After the impact, as in the previous benchmark, the bar stays in contact with the ground during a time $2 \tau_{w}$. During this contact phase, the response of the bar is the superposition of a shock wave due to velocity at the impact and a vibration due to the gravity. When the bar takes off, at time $t_{2}:=t_{1}+2 \tau_{w}$, it has a uniform velocity $v_{f}$ but it is compressed $\left(u\left(x, t_{2}\right)=\tilde{u}(x):=\frac{g_{0}}{c_{0}}\left(x^{2}-2 L x\right)\right)$. Consequently, during the flight phase, the response of the bar, is the superposition of a rigid parabolic motion (due to the gravity and the velocity) and a vibration (due to the initial deformation). If we choose proper parameters (for instance, $\tau_{f}=3 \tau_{w}$ ), we can ensure that the bar reaches the ground with uniform velocity $-v_{f}$ and with displacement field $\tilde{u}$. By doing so, the second impact occurs at time $t_{3}:=t_{2}+2 \tau_{f}=t_{2}+6 \tau_{w}$. When the bar takes off again, at time $t_{4}:=t_{3}+2 \tau_{w}$, it is undeformed and has a uniform velocity $v_{f}$. The next flight phase is a rigid parabolic movement. Then, this sequence of two contact phases and two flight phases repeats periodically. To compute the analytical solution, we use a decomposition on the eigenmodes in addition to the travelling functions. We set $t_{4 k+1}=3 \tau_{w}+16 k \tau_{w}, t_{4 k+2}=t_{4 k+1}+2 \tau_{w}$, 


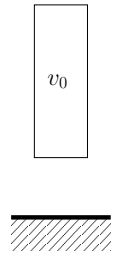

$t=0$

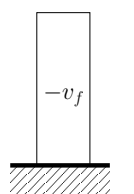

$t_{1}$

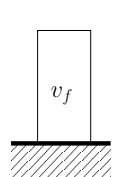

$t_{2}$
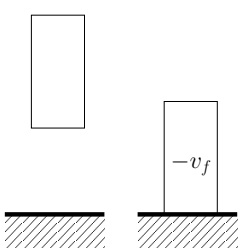

$t_{3}$

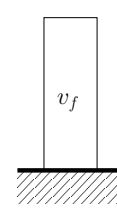

$t_{4}$

Figure 3. Bounces of an elastic bar.

$t_{4 k+2}=t_{4 k+1}+8 \tau_{w}$ and $t_{4 k+4}=t_{4 k+1}+10 \tau_{w}$. We define also the auxiliary functions

$$
\begin{aligned}
& P(x, t)=h_{0}-\frac{1}{2} g_{0}\left(t-\tau_{f}\right)^{2}, \\
& S_{1}(x, t)=\sum_{n=1}^{\infty} a_{n}\left(1-\cos \left(c_{0} \nu_{n} t\right)\right) \sin \left(\nu_{n} x\right), \\
& S_{2}(x, t)=-\frac{2 g_{0} L^{2}}{3 c_{0}^{2}}+\sum_{n=1}^{\infty} b_{n} \cos \left(c_{0} \lambda_{n} t\right) \cos \left(\lambda_{n} x\right),
\end{aligned}
$$

where $a_{n}=\frac{-2 g_{0}}{c_{0}^{2} L \nu_{n}^{3}}, \nu_{n}=\left(n-\frac{1}{2}\right) \frac{\pi}{L}, b_{n}=\frac{4 g_{0}}{g_{0} \lambda_{n}^{2}}, \lambda_{n}=n \frac{\pi}{L}$. The function $S_{1}$ corresponds to the vibration of a bar, clamped at its bottom, initially at rest, under a gravity $g_{0}$. The function $S_{2}$ corresponds to the vibration of a bar, free at its two extremities, with the initial displacement $\tilde{u}$, a zero initial velocity and without external force. The computation of series $S_{1}$ and $S_{2}$ is standard; see [6] for instance. The exact solution is

$$
u(x, t)= \begin{cases}P\left(x, t+\tau_{f}\right) & \text { if } t \leq t_{1} \\ H_{v_{f}}\left(x, t-t_{4 k+1}\right)+S_{1}\left(x, t-t_{4 k+1}\right) & \text { if } t_{4 k+1}<t \leq t_{4 k+2} \\ P\left(x, t-t_{4 k+2}\right)+S_{2}\left(x, t-t_{4 k+2}\right) & \text { if } t_{4 k+2}<t \leq t_{4 k+3} \\ H_{v_{f}}\left(x, t_{4 k+4}-t\right)+S_{1}\left(x, t_{4 k+4}-t\right) & \text { if } t_{4 k+3}<t \leq t_{4 k+4} \\ P\left(x, t-t_{4 k+4}\right) & \text { if } t_{4 k+4}<t \leq t_{4(k+1)+1}\end{cases}
$$

The displacement at the bottom of the bar is represented in Figure 4 (with the parameters chosen in Section 3.3).

\subsection{Numerical simulations}

The parameters used in the numerical simulations are $E=900, \rho=1, L=10, h_{0}=5$. In the first benchmark $v_{0}=10, g_{0}=0$; in the second benchmark $v_{0}=0, g_{0}=10$. The bar is discretized with a uniform meshsize $\Delta x$ and linear finite elements are used. We define $\nu_{c}:=c_{0} \frac{\Delta t}{\Delta x}$ the Courant number, which is the relevant ratio to link the mesh size and the time step. In particular, the central difference scheme with the lumped mass matrix is stable in the linear case under the condition $\nu_{c} \leq 1$. Let us set some notation which will be used in the description of numerical results. If $v$ is a displacement vector, we denote by $v_{0}$ and $(K v)_{0}$ the displacement and the stress at the bottom node of the bar, respectively. In the benchmark problems, the load vector is time-independent; we denote it by $F$. 


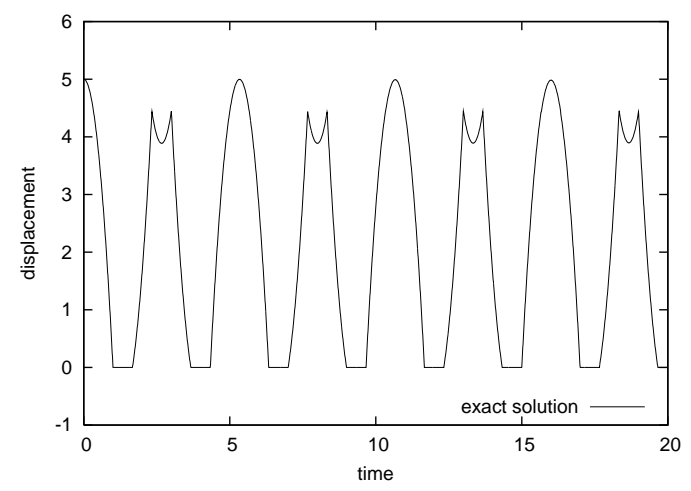

Figure 4. Bounces of an elastic bar. Displacement at the bottom of the bar.

\section{DISCRETIZATIONS WITH EXACT ENFORCEMENT OF THE CONTACT CONDITION}

In this section we combine standard finite elements in space and an exact enforcement of the contact condition at each node of the contact boundary. This leads to the semi-discrete problem,

$$
\begin{aligned}
& M \ddot{u}(t)+K u(t)=F(t)+{ }^{\mathrm{T}} G r(t), \\
& G u(t) \geq 0, \quad r(t) \geq 0, \quad{ }^{\mathrm{T}} r(t) G u(t)=0 .
\end{aligned}
$$

Problem (32)-(33) is a system of differential equations under unilateral constraints. The same kind of formulation arises in rigid-body dynamics with impact [4, 28], so the mathematical results and the numerical methods developed in this framework can in general be applied to our problem. Mathematically, problem (32)-(33) turns out to be delicate. Firstly, the functional framework is not obvious. Due to the unilateral constraints, the velocity can be discontinuous and there is in general no strong solution (i.e. twice differentiable in time) to this problem. We then have to define a concept of weak solution: the displacement $u$ is continuous in time, the velocity $\dot{u}$ is a function with bounded variation in time, the acceleration $\ddot{u}$ and the contact pressure $r$ are measures (they contain impulses). Secondly, this weak solution is in general not unique. Consider the simple example of a point mass impacting a rigid foundation. Before the impact, the motion of the point mass is uniquely determined. After the impact, an infinity of velocities and trajectories are admissible (Figure 5). To recover uniqueness, an additional condition, specifying the velocity after an impact, is needed. If we denote by $v^{-}$the normal velocity before the impact and by $v^{+}$the normal velocity after the impact, we can prescribe

$$
v^{+}=-e v^{-},
$$

where $e$ is a non-negative parameter. This impact law is commonly used in rigid-body dynamics. In our case, it seems reasonable to take $e=0$. Indeed, in the continuous dynamic Signorini problem, the unilateral constraint holds on the boundary and the boundary does not bounce after an impact. In the discrete setting, the unilateral constraint holds on the nodes 


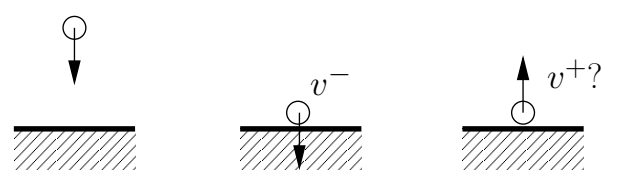

Figure 5. Impact of a point mass.

of the contact boundary. If we want these nodes to have the same behavior as the boundary in the continuous setting, we are led to choose $e=0$. We can now formulate the semi-discrete problem in a rigorous way.

Problem 4.1. Seek a displacement $u:[0, T] \rightarrow \mathbb{R}^{N_{d}}$ and a contact pressure $r:[0, T] \rightarrow \mathbb{R}^{N_{c}}$ such that

$$
\begin{aligned}
& M \ddot{u}+K u=F+{ }^{\mathrm{T}} G r, \\
& G u \geq 0, \quad r \geq 0, \quad{ }^{\mathrm{T}} r G u=0, \\
& { }^{\mathrm{T}} r_{i}(t) G_{i} \dot{u}\left(t^{+}\right)=0 \quad \text { if } \quad G_{i} u(t)=0,
\end{aligned}
$$

with the initial conditions $u(0)=u^{0}$ and $\dot{u}(0)=v^{0}$.

Most of the mathematical terms in equations (35)-(37) must be understood in the sense of measures. In particular ${ }^{\mathrm{T}} r G u$ and ${ }^{\mathrm{T}} r_{i}(t) G_{i} \dot{u}\left(t^{+}\right)$should be defined with suitable duality products. For more details, we refer to $[4,28]$.

Remark 4.1. The impact law is a consequence of the discretization in space. Indeed, the continuous problem does not need an impact law to have a unique solution. This fact is proven in 1D [10, 24]; in higher dimension, the uniqueness is still an open problem, but the difficulty does not seem to come from the absence of an impact law.

Remark 4.2. The semi-discrete solution does not conserve the energy (there is a loss of energy at each impact of a node). This is another difference with the continuous solution.

Remark 4.3. The impact law is different from the concept of persistency condition sometimes encountered in the literature [1, 21, 22, 23]. The persistency condition is defined in the continuous setting and in the fully discrete setting. It requires that the contact force does not work. Note that in the continuous problem, the persistency condition seems to be a consequence of the Signorini condition (it is at least proven in 1D).

\subsection{Implicit schemes}

To motivate the discussion, let us begin with an ill-founded discretization. We choose a Newmark scheme (trapezoidal rule) for the elastodynamic part and we enforce the contact condition (36) at a certain time, say $t^{n+1}$. We pay no attention to the impact law (37). This choice corresponds to Discretization 4.1 with $\alpha=0, \beta=1 / 4, \gamma=1 / 2$.

Discretization 4.1 (HHT-Newmark) Seek $u^{n+1}, \dot{u}^{n+1}, \ddot{u}^{n+1} \in \mathbb{R}^{N_{d}}$, and $r^{n+1} \in \mathbb{R}^{N_{c}}$ 
such that

$$
\begin{aligned}
& M \ddot{u}^{n+1}+K u^{n+1+\alpha}=F^{n+1+\alpha}+{ }^{\mathrm{T}} G r^{n+1}, \\
& G u^{n+1} \geq 0, \quad r^{n+1} \geq 0, \quad{ }^{\mathrm{T}} r^{n+1} G u^{n+1}=0, \\
& u^{n+1}=u^{n}+\Delta t \dot{u}^{n}+\frac{\Delta t^{2}}{2} \ddot{u}^{n+2 \beta}, \\
& \dot{u}^{n+1}=\dot{u}^{n}+\Delta t \ddot{u}^{n+\gamma} .
\end{aligned}
$$

At each time step, Problem (38)-(41) is equivalent to a linear complementarity problem and is well-posed. In contrast to the static case, the matrix $K$ does not need to be definite for the problem to be well-posed (Dirichlet boundary conditions are not needed). When this scheme is tested on the first benchmark, we observe large spurious oscillations on the contact pressure and small spurious oscillations on the displacement during the contact phase (Figure 6). On the second benchmark, we observe a poor behavior in long-time and a poor energy conservation (Figure 7). Let us try to explain what happens exactly. Suppose there is contact at the $i$ th node of the contact boundary at time $t^{n+1}$ (i.e. $G_{i} u^{n+1}=0$ ), then

$$
\begin{aligned}
G_{i} \dot{u}^{n+1} & =-\frac{1}{2 \Delta t} G_{i} u^{n}+\left(1-\frac{\beta}{\gamma}\right) G_{i} \dot{u}^{n}+\Delta t \frac{2 \beta-\gamma}{2 \beta} G_{i} \ddot{u}^{n}, \\
G_{i} \ddot{u}^{n+1} & =-\frac{1}{\beta \Delta t^{2}} G_{i} u^{n}-\frac{1}{\beta \Delta t} G_{i} \dot{u}^{n}-\frac{1-2 \beta}{2 \beta} G_{i} \ddot{u}^{n} .
\end{aligned}
$$

As we can see, the impact law is not satisfied since we would expect that after an impact, $G_{i} \dot{u}^{n}=G_{i} \ddot{u}^{n}=0$. During a contact phase following an impact, the velocity and the acceleration oscillate. For the acceleration, the magnitude of the oscillations is $\frac{v_{i}}{\Delta t}$, where $v_{i}$ is the velocity before the impact. These oscillations of the acceleration trigger oscillations of magnitude $\frac{m_{i} v_{i}}{\Delta t}$ on the contact pressure, where $m_{i}$ is the mass associated with the node $i$ (Figure 6). Moreover, the energy balance takes the form,

$$
\mathcal{E}^{n+1}-\mathcal{E}^{n}={ }^{\mathrm{T}}\left(\frac{r^{n+1}+r^{n}}{2}\right)\left(G u^{n+1}-G u^{n}\right)+{ }^{\mathrm{T}}\left(\frac{F^{n+1}+F^{n}}{2}\right)\left(u^{n+1}-u^{n}\right),
$$

so that the contact force works when a node changes status. When a node comes into contact $\left(G_{i} u^{n}>0, r^{n}=0, G_{i} u^{n+1}=0, r^{n+1}>0\right)$, the work is negative; when a node is released $\left(G_{i} u^{n}=0, r^{n}>0, G_{i} u^{n+1}>0, r^{n+1}=0\right)$, the work is positive. As the contact pressure is polluted by large oscillations, this strongly perturbs the rest of the structure (Figure 7). The poor behavior of the Newmark scheme can be summarized by the following diagram:

(a) large oscillations of the acceleration at the contact boundary

$\downarrow$

(b) large oscillations of the contact pressure

$\downarrow$

(c) perturbation of the whole structure

In themselves the oscillations of the acceleration at the contact boundary are not a problem. The oscillations of the contact pressure are more troublesome if a Lagrangian method is used for solving the linear complementarity problem at each time step (the Lagrange multiplier being equal to the contact pressure). Of course, the perturbation of the whole structure must 

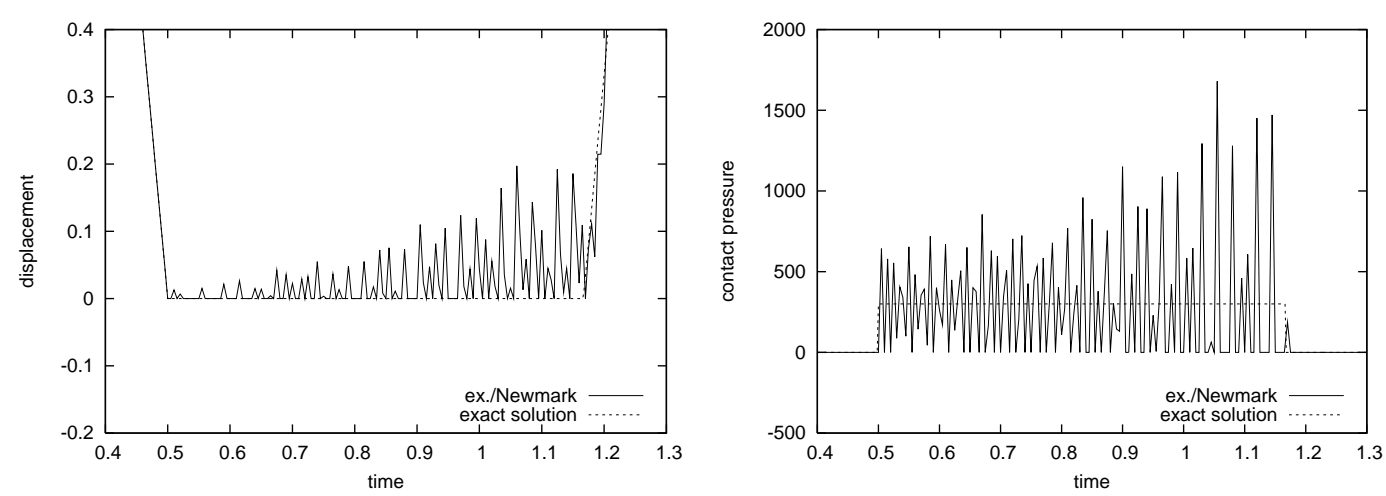

Figure 6. Impact of an elastic bar. Displacement $u_{0}^{n}$ (left) and contact pressure $r^{n}$ (right). Discretization 4.1 with $\alpha=0, \beta=1 / 4$ and $\gamma=1 / 2 . \Delta x=0.1, \Delta t=0.005, \nu_{c}=1.5$.
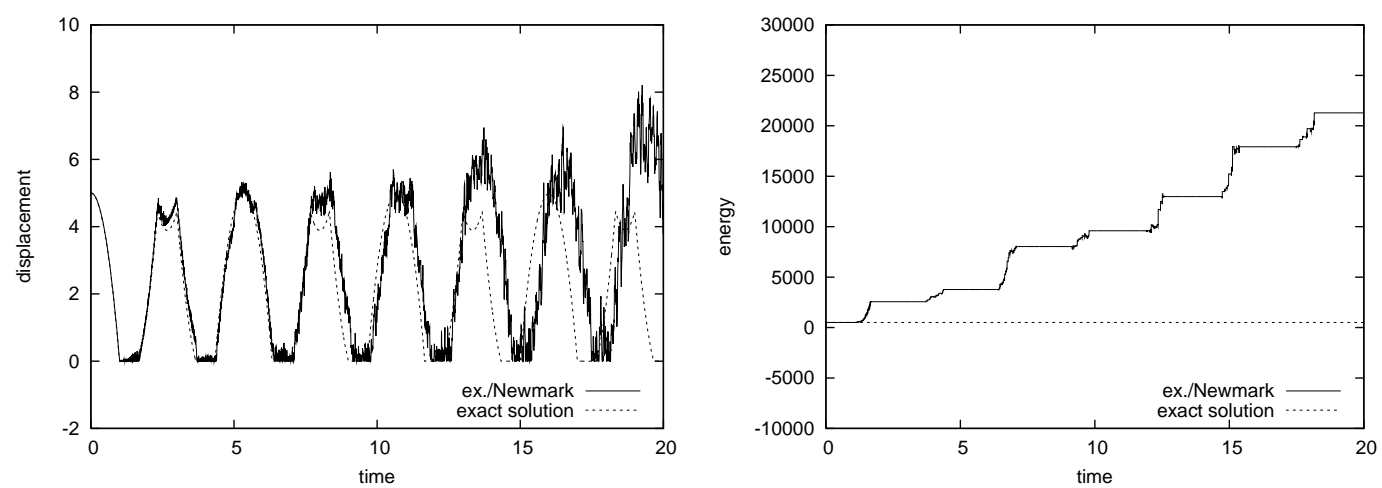

Figure 7. Bounces of an elastic bar. Displacement $u_{0}^{n}$ (left) and energy $\mathcal{E}^{n}-{ }^{\mathrm{T}} F u^{n}$ (right). Discretization 4.1 with $\alpha=0, \beta=1 / 4$ and $\gamma=1 / 2 . \Delta x=0.1, \Delta t=0.005, \nu_{c}=1.5$.

be avoided. Several options can be considered to design better algorithms. The first option consists in using dissipative schemes, such as HHT schemes (Discretization 4.1). The spurious oscillations are then damped (Figure 8), but at the expense of poor energy conservation (Figure 9 ). First-order schemes like $\theta$-schemes, which are implicit, unconditionally stable, dissipative schemes, yield the same kind of results (Discretization 4.2).

Discretization $4.2\left(\theta\right.$-schemes [30]) Seek $u^{n+1}, \dot{u}^{n+1} \in \mathbb{R}^{N_{d}}$, and $r^{n+1} \in \mathbb{R}^{N_{c}}$ such that

$$
\begin{aligned}
& M \ddot{u}^{n+\frac{1}{2}}+K u^{n+\theta}=F^{n+\theta}+{ }^{\mathrm{T}} G r^{n+1}, \\
& G u^{n+1} \geq 0, \quad r^{n+1} \geq 0, \quad{ }^{\mathrm{T}} r^{n+1} G u^{n+1}=0, \\
& u^{n+1}=u^{n}+\Delta t \dot{u}^{n+\theta}, \\
& \dot{u}^{n+1}=\dot{u}^{n}+\Delta t \ddot{u}^{n+\frac{1}{2}} .
\end{aligned}
$$

Remark 4.4. It is sometimes advocated in the literature that first-order schemes must be 

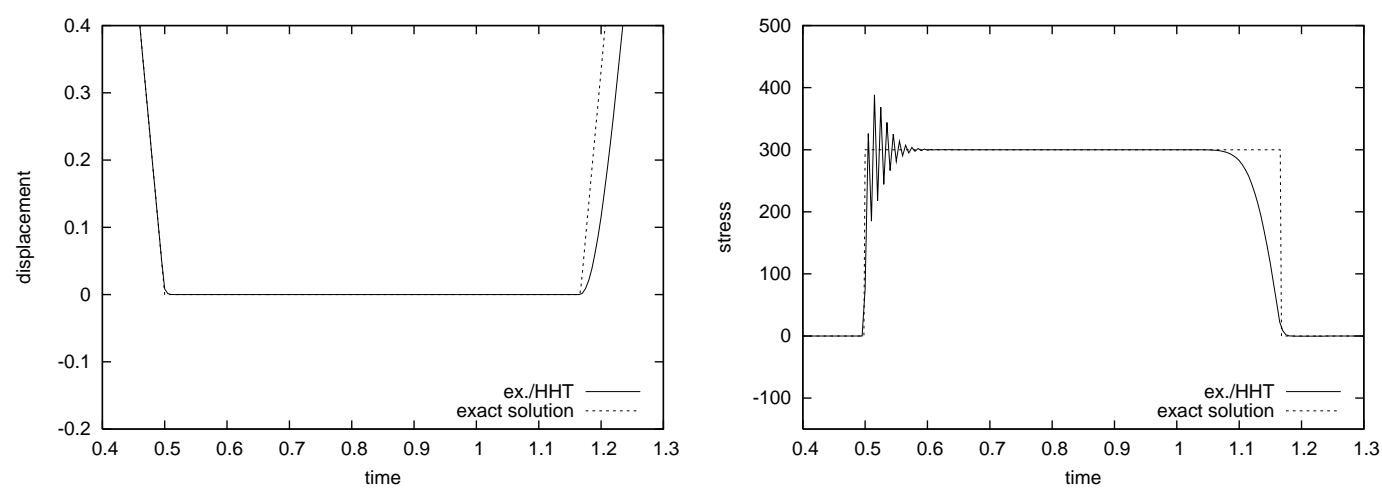

Figure 8. Impact of an elastic bar. Displacement $u_{0}^{n}$ (left) and contact pressure $r^{n}$ (right) Discretization 4.1 with $\alpha=-0.2, \beta=1 / 4(1-\alpha)^{2}, \gamma=1 / 2-\alpha . \Delta x=0.1, \Delta t=0.005, \nu_{c}=1.5$.
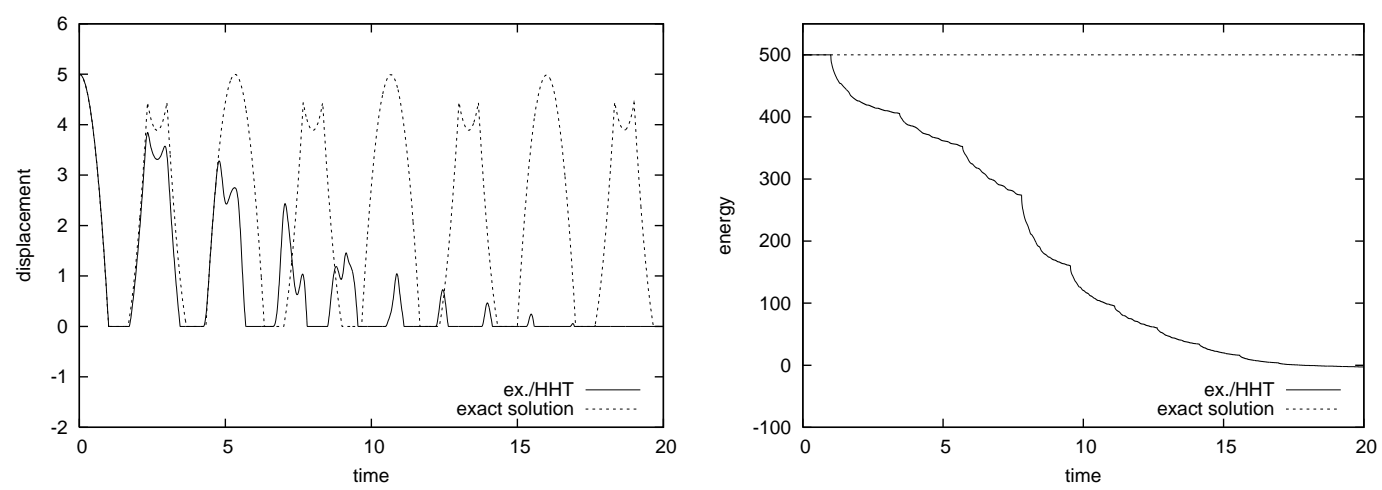

Figure 9. Bounces of an elastic bar. Displacement $u_{0}^{n}$ (left) and energy $\mathcal{E}^{n}-{ }^{\mathrm{T}} F u^{n}$ (right). Discretization 4.1 with $\alpha=-0.2, \beta=1 / 4(1-\alpha)^{2}, \gamma=1 / 2-\alpha . \Delta x=0.1, \Delta t=0.005, \nu_{c}=1.5$.

preferred to second-order schemes for dynamic contact problems, due to the non-smoothness of the solution. We must distinguish two issues: the treatment of the contact condition and the treatment of the shock waves induced by the contact. As discussed previously, a proper treatment of the contact condition is not related to the order of the scheme. As for the shock waves, they require a scheme with dissipation and there exist second-order schemes with dissipation, such as the HHT or Chung-Hulbert schemes. We refer to the Appendix for further discussion.

A second option consists in finding a scheme which satisfies the impact law or, more precisely, a scheme which enforces the acceleration to be zero during the contact phases. No implicit Newmark scheme achieves this. An extra-step is needed to enforce the impact law (Discretization 4.3).

\section{Discretization 4.3 (naive stabilized Newmark)}

1. Seek $u^{n+1} \in \mathbb{R}^{N_{d}}, \dot{u}^{n+1} \in \mathbb{R}^{N_{d}}, \ddot{u}^{n+1} \in \mathbb{R}^{N_{d}}$, and $r^{n+1} \in \mathbb{R}^{N_{c}}$ satisfying (38)-(41). 
2. If $G_{i} u^{n}<0$ and $G_{i} u^{n+1}=0$, then $\dot{u}^{n+1}$ and $\ddot{u}^{n+1}$ are modified so that

$$
G_{i} \dot{u}^{n+1}=0 \quad \text { and } \quad G_{i} \ddot{u}^{n+1}=0 .
$$
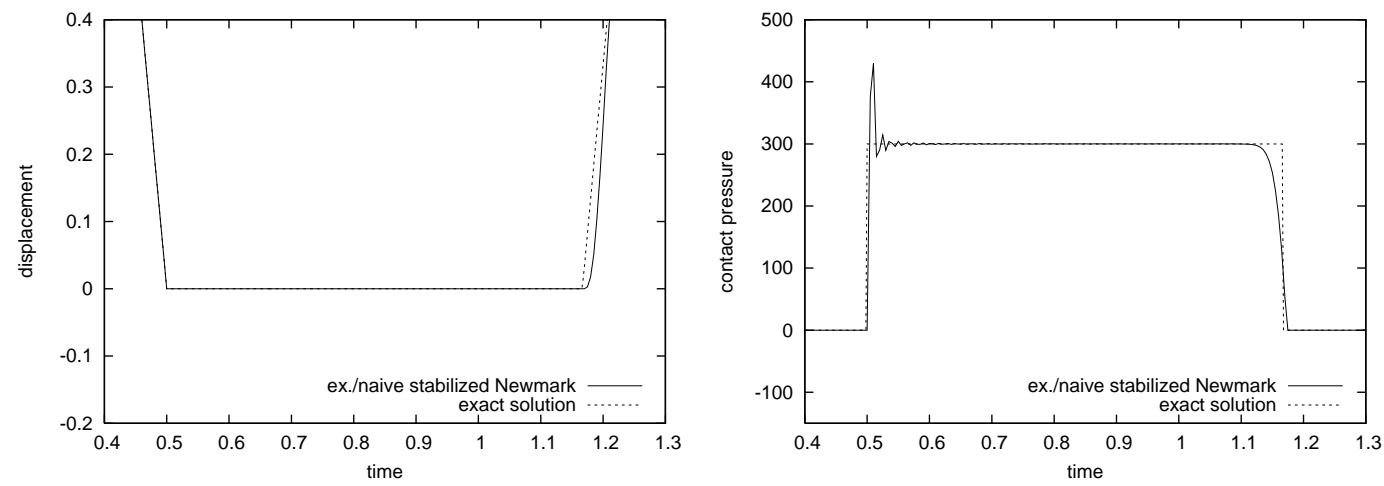

Figure 10. Impact of an elastic bar. Displacement $u_{0}^{n}$ (left) and contact pressure $r^{n}$ (right). Discretization 4.3 with $\alpha=0, \beta=1 / 4$ and $\gamma=1 / 2 . \Delta x=0.1, \Delta t=0.005, \nu_{c}=1.5$.

As illustrated in Figure 10, the large spurious oscillations have disappeared. However, this stabilization takes effect only one step after the impact, which explains the peak in the contact pressure just after the impact. Stabilizations procedures which enforce the impact law as soon as the impact occurs have been proposed in [29] and [7]. This second problem will be presented at the end of the section (Discretization 4.6). As a third option, it is possible to prevent the oscillations of the acceleration from transferring to the contact pressure by removing the mass at the contact boundary. This approach will be developed in Section 7 . The fourth option consists in finding a time discretization where the contact force does not work or is at least dissipative. For instance, the midpoint scheme with an enforcement of the contact condition at time $t^{n+1}$ achieves the following energy balance,

$$
\mathcal{E}^{n+1}-\mathcal{E}^{n}={ }^{\mathrm{T}} r^{n+1}\left(G u^{n+1}-G u^{n}\right)+{ }^{\mathrm{T}} F^{n+\frac{1}{2}}\left(u^{n+1}-u^{n}\right) .
$$

It is easy to check that the work of the contact force is always non-positive. As illustrated in Figure 11, the contact pressure still oscillates but the stress is practically free of oscillations. However, the losses of energy are quite important and deteriorate the long-time behavior (Figure 12).

Discretization 4.4 (Midpoint-implicit contact) Seek $u^{n+1}$, $\dot{u}^{n+1} \in \mathbb{R}^{N_{d}}$, and $r^{n+1} \in$ $\mathbb{R}^{N_{c}}$ such that

$$
\begin{aligned}
& M \ddot{u}^{n+\frac{1}{2}}+K u^{n+\frac{1}{2}}=F^{n+\frac{1}{2}}+{ }^{\mathrm{T}} G r^{n+1}, \\
& G u^{n+1} \geq 0, \quad r^{n+1} \geq 0, \quad{ }^{\mathrm{T}} r^{n+1} G u^{n+1}=0, \\
& u^{n+1}=u^{n}+\Delta t \dot{u}^{n+\frac{1}{2}}, \\
& \dot{u}^{n+1}=\dot{u}^{n}+\Delta t \ddot{u}^{n+\frac{1}{2}} .
\end{aligned}
$$


Another scheme with dissipative contact has been proposed in [17]. The Newmark scheme with parameters $\beta=1 / 2$ and $\gamma=1$ and with an enforcement of the contact condition at time $t^{n+1}$ yields the following energy balance

$$
\mathcal{E}^{n+1}-\mathcal{E}^{n}={ }^{\mathrm{T}} r^{n+1}\left(G u^{n+1}-G u^{n}\right)-\frac{1}{2} \mathrm{~T}\left(u^{n+1}-u^{n}\right) K\left(u^{n+1}-u^{n}\right)+{ }^{\mathrm{T}} F^{n+1}\left(u^{n+1}-u^{n}\right) .
$$

The work of the contact force is always non-positive, but there is a strong bulk dissipation. To remove this dissipation, one can, as proposed in [17], discretize the acceleration coming from the contact forces with the dissipative parameters $(\beta=1 / 2$ and $\gamma=1)$ and the acceleration coming from the elastic forces with a trapezoidal rule $(\beta=1 / 4$ and $\gamma=1 / 2)$. This yields Discretization 4.5. With such a discretization, the energy balance is

$$
\mathcal{E}^{n+1}-\mathcal{E}^{n}={ }^{\mathrm{T}} r^{n+1}\left(G u^{n+1}-G u^{n}\right)+{ }^{\mathrm{T}} F^{n+1}\left(u^{n+1}-u^{n}\right) .
$$

The numerical results are similar to those obtained with Discretization 4.4.

Discretization 4.5 (Newmark with dissipative contact [17]) Seek $u^{n+1}, \dot{u}^{n+1}, \ddot{u}_{i n t}^{n+1}$, $\ddot{u}_{\text {con }}^{n+1} \in \mathbb{R}^{N_{d}}$, and $r^{n+1} \in \mathbb{R}^{N_{c}}$ such that

$$
\begin{aligned}
& M \ddot{u}^{n+1}+K u^{n+1}=F^{n+1}+{ }^{\mathrm{T}} G r^{n+1}, \\
& G u^{n+1} \geq 0, \quad r^{n+1} \geq 0, \quad{ }^{\mathrm{T}} r^{n+1} G u^{n+1}=0, \\
& u^{n+1}=u^{n}+\Delta t \dot{u}^{n}+\frac{\Delta t^{2}}{2} \ddot{u}_{i n t}^{n+2 \beta}+\frac{\Delta t^{2}}{2} \ddot{u}_{c o n}^{n+1}, \\
& \dot{u}^{n+1}=\dot{u}^{n}+\Delta t \ddot{u}_{\text {int }}^{n+\gamma}+\Delta t \ddot{u}_{c o n}^{n+1},
\end{aligned}
$$

where $\ddot{u}^{n+1}=\ddot{u}_{\text {int }}^{n+1}+\ddot{u}_{c o n}^{n+1}$ and $M \ddot{u}_{c o n}^{n+1}={ }^{\mathrm{T}} G r^{n+1}$.

To compensate the losses of energy in schemes with dissipative contact, the so-called velocityupdate method has been introduced in [23]. Applied to Discretization 4.4, this procedure does not improve significantly the long-time behavior on our benchmark.

In [7], the authors add to Discretization 4.5 a stabilization procedure (Discretization 4.6).
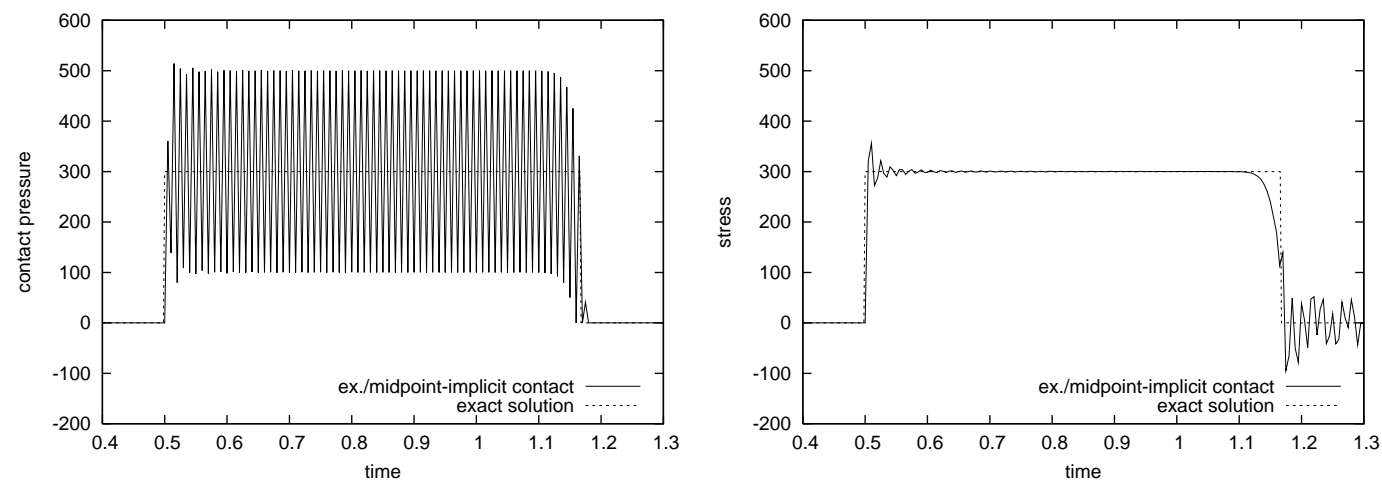

Figure 11. Impact of an elastic bar. Contact pressure $r^{n}$ (left) and stress $\left(K u^{n}\right)_{0}$ (right). Discretization 4.4. $\Delta x=0.1, \Delta t=0.005, \nu_{c}=1.5$. 

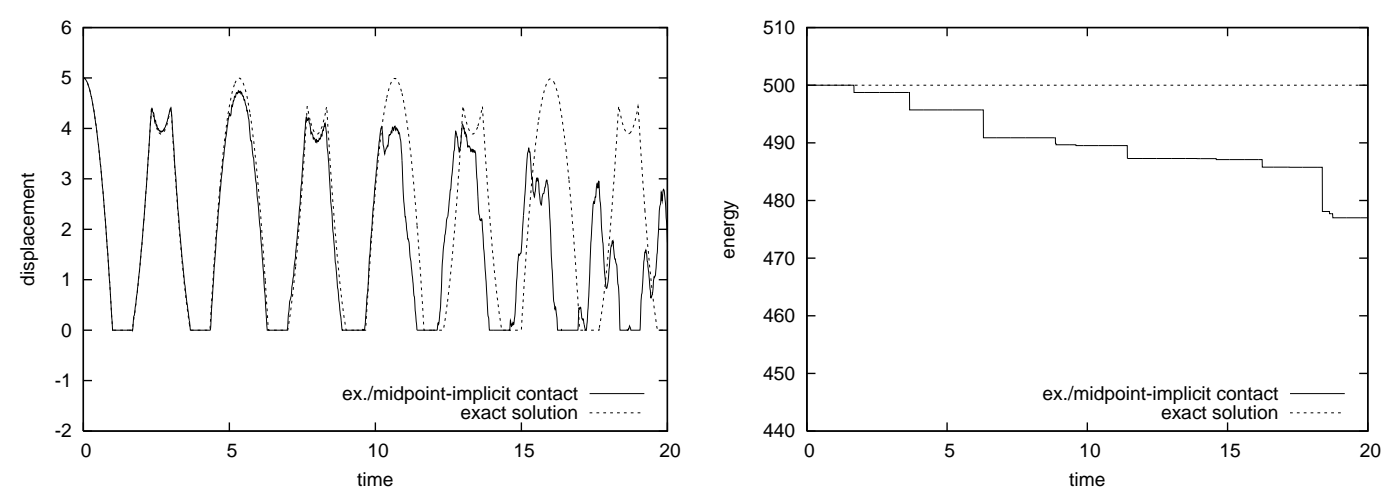

Figure 12. Bounces of an elastic bar. Displacement $u_{0}^{n}$ (left) and energy $\mathcal{E}^{n}-{ }^{\mathrm{T}} F u^{n}$ (right). Discretization 4.4. $\Delta x=0.1, \Delta t=0.005, \nu_{c}=1.5$.

Discretization 4.6 (stabilized Newmark [7])

1. Seek $u_{\text {pred }}^{n+1} \in \mathbb{R}^{N_{d}}$ and $\lambda^{n+1} \in \mathbb{R}^{N_{c}}$ such that

$$
\begin{aligned}
& M u_{\text {pred }}^{n+1}=M u^{n}+\Delta t M \dot{u}^{n}, \\
& G u_{\text {pred }}^{n+1} \geq 0, \quad \lambda^{n+1} \geq 0, \quad{ }^{\mathrm{T}} \lambda^{n+1} G u_{\text {pred }}^{n+1}=0 .
\end{aligned}
$$

2. Seek $u^{n+1}, \dot{u}^{n+1}, \ddot{u}_{\text {int }}^{n+1}, \ddot{u}_{\text {con }}^{n+1} \in \mathbb{R}^{N_{d}}$, and $r^{n+1} \in \mathbb{R}^{N_{c}}$ such that

$$
\begin{aligned}
& M \ddot{u}^{n+1}+K u^{n+1}=F^{n+1}+{ }^{\mathrm{T}} G r^{n+1}, \\
& G u^{n+1} \geq 0, \quad r^{n+1} \geq 0, \quad \quad{ }^{\mathrm{T}} r^{n+1} G u^{n+1}=0, \\
& u^{n+1}=u_{\text {pred }}^{n+1}+\frac{\Delta t^{2}}{2} \ddot{u}_{i n t}^{n+2 \beta}+\frac{\Delta t^{2}}{2} \ddot{u}_{c o n}^{n+1}, \\
& \dot{u}^{n+1}=\dot{u}^{n}+\Delta t \ddot{u}_{\text {int }}^{n+\gamma}+\Delta t \ddot{u}_{\text {con }}^{n+1},
\end{aligned}
$$

where $\ddot{u}^{n+1}=\ddot{u}_{i n t}^{n+1}+\ddot{u}_{c o n}^{n+1}$ and $M \ddot{u}_{c o n}^{n+1}={ }^{\mathrm{T}} G r^{n+1}$.

With this scheme, the contact pressure is now almost free of oscillations (Figure 13). Indeed, if $G_{i} u^{n+1}=G_{i} u_{\text {pred }}^{n+1}=0$, then

$$
G_{i} \ddot{u}_{i n t}^{n+2 \beta}+G_{i} \ddot{u}_{c o n}^{n+1}=0 .
$$

The long-time behavior is still poor (Figure 14). The additional step required by this scheme is not expensive compared with the main step, especially if the mass matrix is lumped.

\subsection{Semi-explicit schemes}

Now, we try to discretize the elastodynamic part of the problem with an explicit scheme, such as the central difference scheme. It is not possible to enforce an explicit contact condition. The contact condition can be enforced implicitly (Discretization 4.7) or by a projection step (Discretization 4.8). 

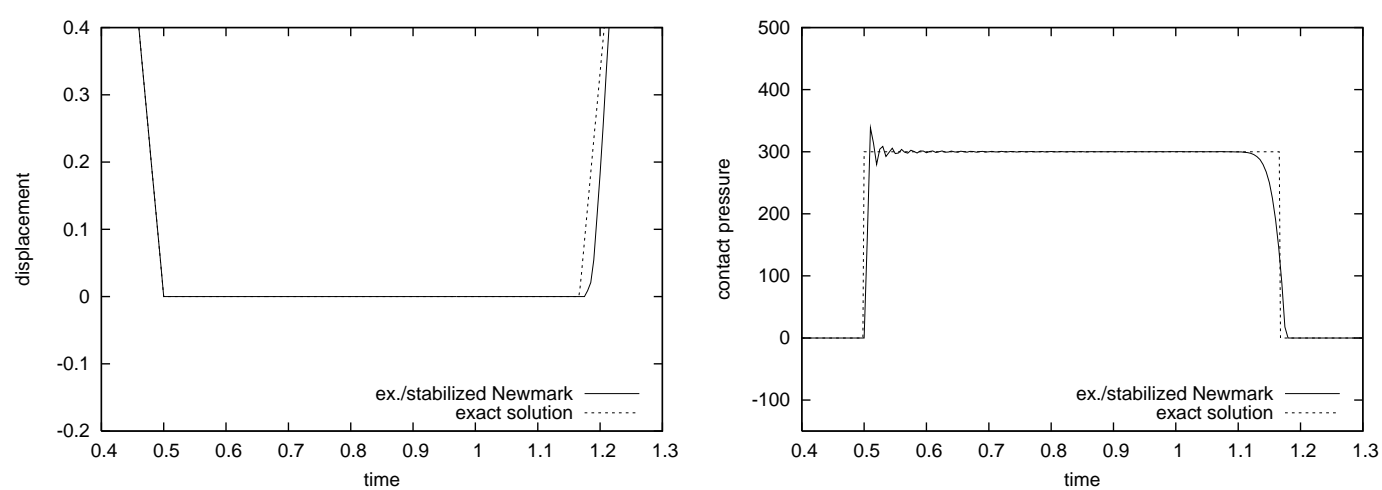

Figure 13. Impact of an elastic bar. Displacement $u_{0}^{n}$ (left) and contact pressure $r^{n}$ (right). Discretization 4.6 with $\beta=1 / 4$ and $\gamma=1 / 2$ (lumped mass matrix). $\Delta x=0.1, \Delta t=0.005, \nu_{c}=1.5$.
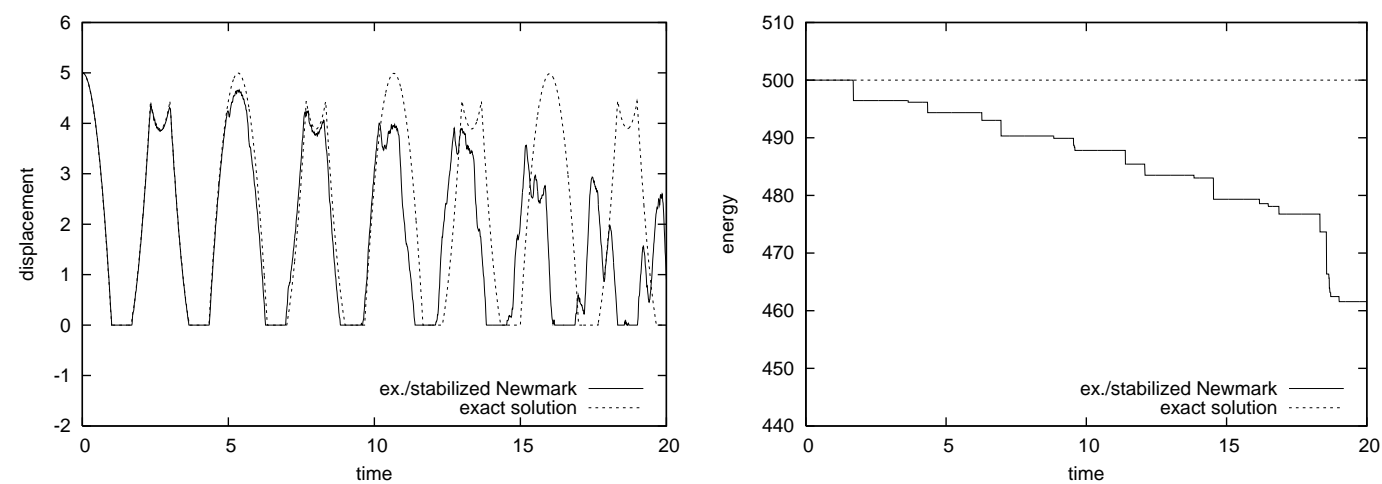

Figure 14. Bounces of an elastic bar. Displacement $u_{0}^{n}$ (left) and energy $\mathcal{E}^{n}-{ }^{\mathrm{T}} F u^{n}$ (right). Discretization 4.6 with $\beta=1 / 4$ and $\gamma=1 / 2$ (lumped mass matrix). $\Delta x=0.1, \Delta t=0.005, \nu_{c}=1.5$.

Discretization 4.7 (Central differences-implicit contact $[25,26]$ ) Seek $u^{n+1} \in \mathbb{R}^{N_{d}}$ and $r^{n+1} \in \mathbb{R}^{N_{c}}$ such that

$$
\begin{aligned}
& M\left(\frac{u^{n+1}-2 u^{n}+u^{n-1}}{\Delta t^{2}}\right)+K u^{n}=F^{n}+{ }^{\mathrm{T}} G r^{n+1}, \\
& G u^{n+1} \geq 0, \quad r^{n+1} \geq 0, \quad{ }^{\mathrm{T}} r^{n+1} G u^{n+1}=0 .
\end{aligned}
$$

Discretization 4.8 (Central differences-projected contact [5])

1. Seek $u_{\text {pred }}^{n+1} \in \mathbb{R}^{N_{d}}$ such that

$$
M\left(\frac{u_{\text {pred }}^{n+1}-2 u^{n}+u^{n-1}}{\Delta t^{2}}\right)+K u^{n}=F^{n} .
$$


2. Seek $u^{n+1} \in \mathbb{R}^{N_{d}}$ and $r^{n+1} \in \mathbb{R}^{N_{c}}$ such that

$$
\begin{aligned}
& u^{n+1}=u_{\text {pred }}^{n+1}+{ }^{\mathrm{T}} G r^{n+1}, \\
& G u^{n+1} \geq 0, \quad r^{n+1} \geq 0, \quad{ }^{\mathrm{T}} r^{n+1} G u^{n+1}=0 .
\end{aligned}
$$

The second approach seems more explicit, but, actually, the two approaches are equivalent when the mass matrix $M$ is diagonal. Discretization 4.7 has been proposed in [27] in 1D and $[25,26]$ in the framework of rigid-body dynamics. Discretization 4.7 has been proposed in [5]. It is easy to check that the acceleration at the contact boundary vanishes during a contact phase. Indeed, if $G_{i} u^{n+1}=G_{i} u^{n}=G_{i} u^{n-1}=0$, then

$$
G_{i} \ddot{u}^{n}=G_{i}\left(\frac{u^{n+1}-2 u^{n}+u^{n-1}}{\Delta t^{2}}\right)=0 .
$$

Consequently, there are no spurious oscillations (Figure 15). The energy balance reads

$$
\mathcal{E}_{0, \frac{1}{2}}^{n+1}-\mathcal{E}_{0, \frac{1}{2}}^{n}={ }^{\mathrm{T}}\left(\frac{r^{n+2}+r^{n+1}}{2}\right)\left(G u^{n+1}-G u^{n}\right)+{ }^{\mathrm{T}}\left(\frac{F^{n+1}+F^{n}}{2}\right)\left(u^{n+1}-u^{n}\right) .
$$

The losses of energy are quite important (Figure 16). In 1D, the convergence of the discrete solutions to the continuous solution, provided a stability condition is met (the same as in the linear case), has been established in [27]. The convergence of the discrete solutions to a semi-discrete solution has been proven in $[25,26]$.
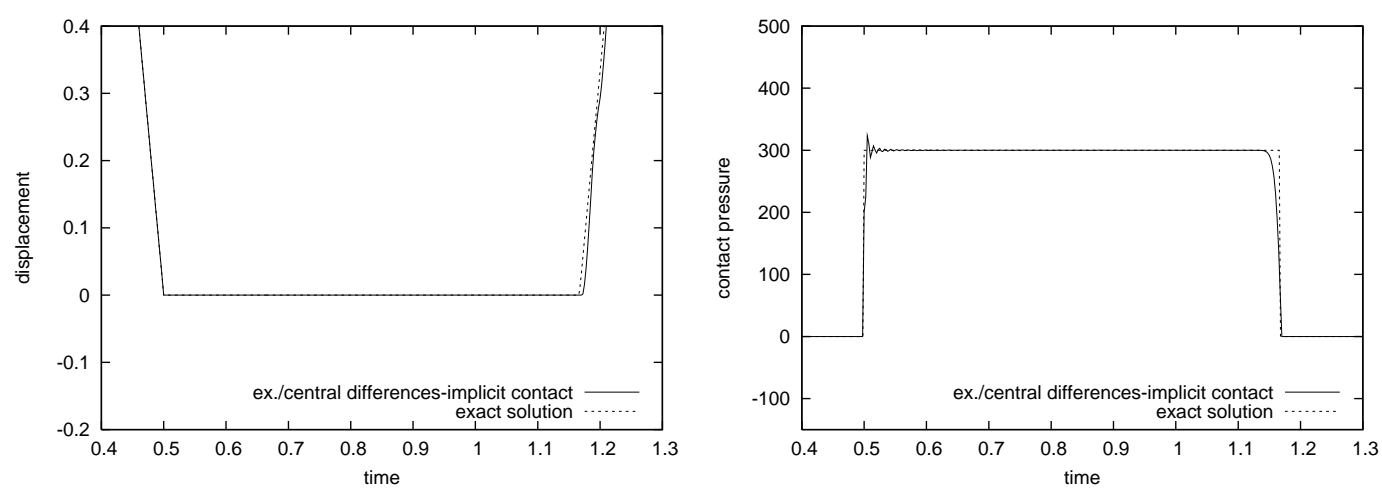

Figure 15. Impact of an elastic bar. Displacement $u_{0}^{n}$ (left) and contact pressure $r^{n}$ (right). Discretization 4.7 (lumped mass matrix). $\Delta x=0.1, \Delta t=0.0025, \nu_{c}=0.75$.

\section{DISCRETIZATIONS WITH PENALTY CONTACT CONDITION}

In this part we combine standard finite elements in space and a penalty approximation of the contact condition. Then, the semi-discrete problem is a mere system of ODEs.

Problem 5.1. Seek a displacement $u:[0, T] \rightarrow \mathbb{R}^{N_{d}}$ such that, $\forall t \in[0, T]$,

$$
M \ddot{u}(t)+K u(t)=f(t)+{ }^{\mathrm{T}} G R_{\epsilon}(G u(t)),
$$

with the initial conditions $u(0)=u^{0}$ and $\dot{u}(0)=v^{0}$. 

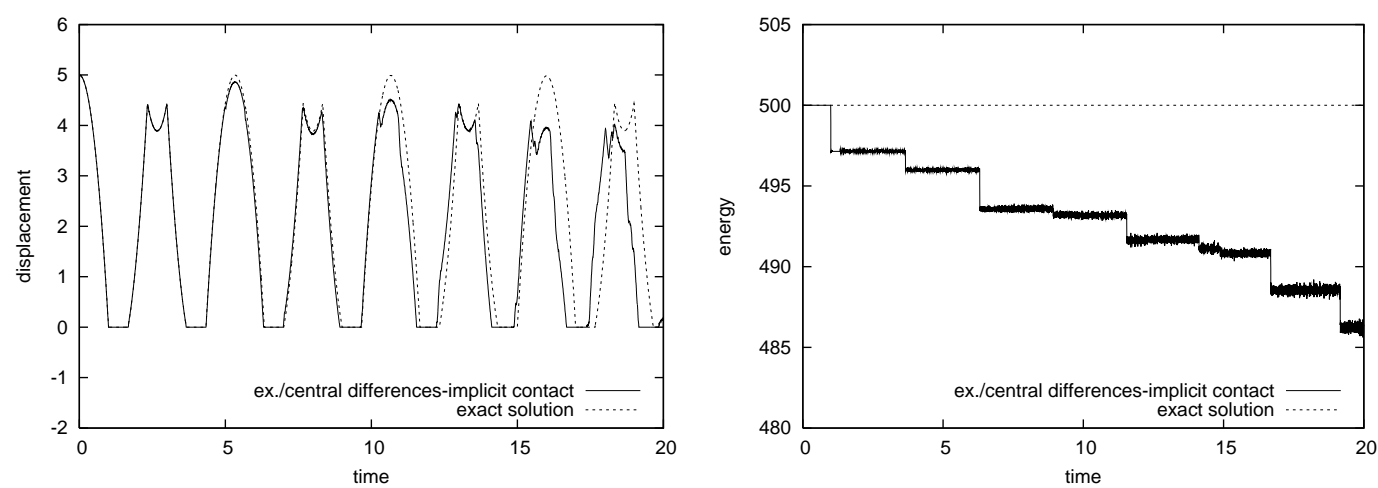

Figure 16. Bounces of an elastic bar. Displacement $u_{0}^{n}$ (left) and energy $\mathcal{E}^{n}-{ }^{\mathrm{T}} F u^{n}$ (right). Discretization 4.7 (lumped mass matrix). $\Delta x=0.1, \Delta t=0.0025, \nu_{c}=0.75$.

Problem 5.1, being a system of ODEs, has one and only one solution, which is furthermore twice differentiable in time.

\subsection{Implicit schemes}

To begin with, we discretize Problem 5.1 with an implicit Newmark scheme.

Discretization 5.1 (Newmark) Seek $u^{n+1}, \dot{u}^{n+1}, \ddot{u}^{n+1} \in \mathbb{R}^{N_{d}}$, such that

$$
\begin{aligned}
& M \ddot{u}^{n+1}+K u^{n+1}=F^{n+1}+{ }^{\mathrm{T}} G R_{\epsilon}\left(G u^{n+1}\right), \\
& u^{n+1}=u^{n}+\Delta t \dot{u}^{n}+\frac{\Delta t^{2}}{2} \ddot{u}^{n+2 \beta}, \\
& \dot{u}^{n+1}=\dot{u}^{n}+\Delta t \ddot{u}^{n+\gamma} .
\end{aligned}
$$

We observe that the penalty formulation tends to reduce spurious oscillations (Figure 17). Nevertheless, the oscillations grow with the penalty parameter $1 / \epsilon$ (Figure 18). This is not surprising since the penalty contact condition tends to the exact contact condition when $1 / \epsilon$ tends to infinity. If the oscillations are too large, stabilization procedures can be used (see for instance the procedure described in [1]). With the addition of a penalty term, the Newmark scheme (trapezoidal rule) no longer conserves the energy (Figure 19). In [1, 14], the authors proposed a discretization of the penalty term which enables to recover energy conservation (Discretization 5.2). It is based on a midpoint scheme. On our benchmark problems, it does not yield significantly better results.

Discretization 5.2 (Energy-conserving midpoint $[1,14]$ ) Seek $u^{n+1}$, $\dot{u}^{n+1} \in \mathbb{R}^{N_{d}}$, such that

$$
\begin{aligned}
& M \ddot{u}^{n+\frac{1}{2}}+K u^{n+\frac{1}{2}}=F^{n+\frac{1}{2}}+{ }^{\mathrm{T}} G \tilde{R}_{\epsilon}\left(G u^{n+1}, G u^{n}\right), \\
& u^{n+1}=u^{n}+\Delta t \dot{u}^{n+\frac{1}{2}}, \\
& \dot{u}^{n+1}=\dot{u}^{n}+\Delta t \ddot{u}^{n+\frac{1}{2}},
\end{aligned}
$$



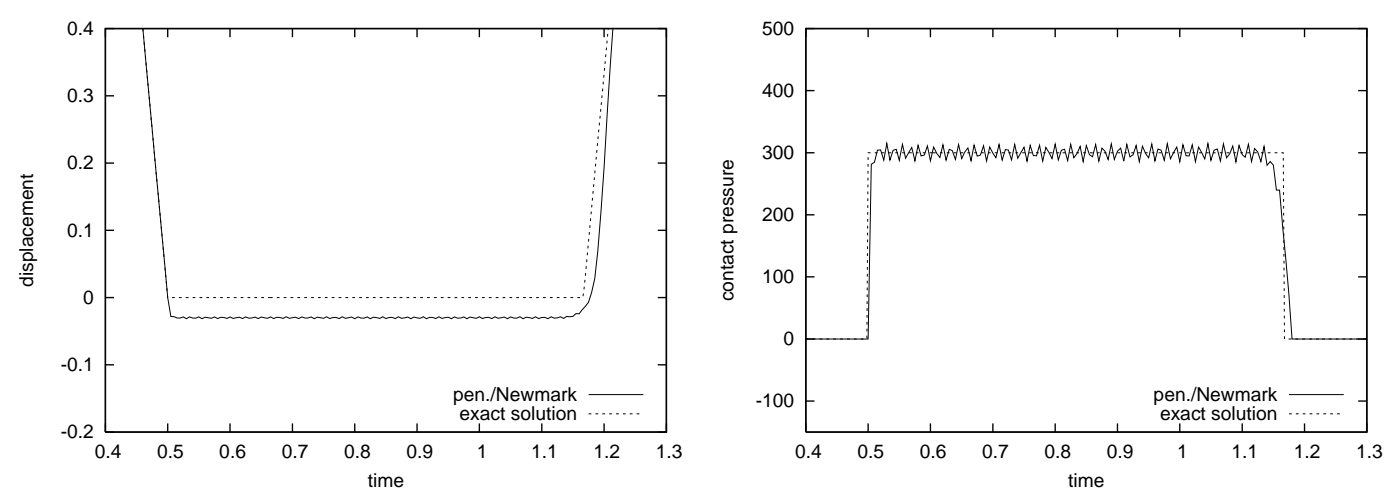

Figure 17. Impact of an elastic bar. Displacement $u_{0}^{n}$ (left) and contact pressure ${ }^{\mathrm{T}} G R_{\epsilon}\left(G u^{n}\right)$ (right). Discretization 5.1 with $\alpha=0, \beta=1 / 4, \gamma=1 / 2, \epsilon=10^{-4} . \Delta x=0.1, \Delta t=0.005, \nu_{c}=1.5$.
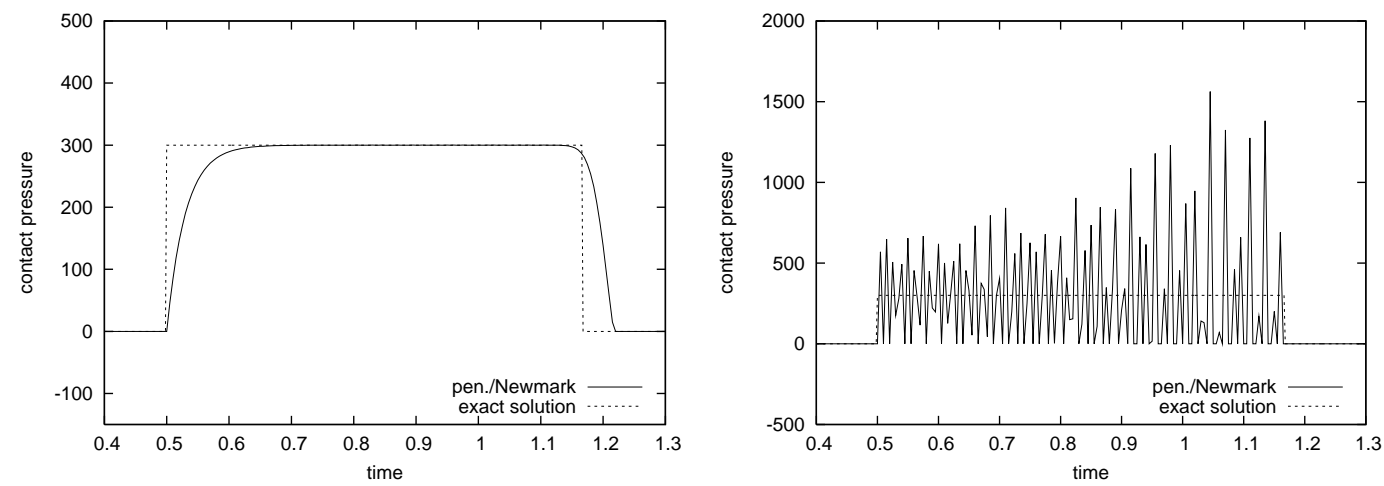

Figure 18. Impact of an elastic bar. Contact pressure ${ }^{\mathrm{T}} G R_{\epsilon}\left(G u^{n}\right)$. Discretization 5.1 with $\alpha=0$, $\beta=1 / 4$ and $\gamma=1 / 2 . \Delta x=0.1, \Delta t=0.005, \nu_{c}=1.5 . \epsilon=10^{-3}$ (left) and $\epsilon=10^{-5}$ (right).

where

$$
\left(\tilde{R}_{\epsilon}\left(G u^{n+1}, G u^{n}\right)\right)_{i}= \begin{cases}\frac{1}{2 \epsilon} \frac{\left(\left(G_{i} u^{n+1}\right)^{-}\right)^{2}-\left(\left(G_{i} u^{n}\right)^{-}\right)^{2}}{G_{i} u^{n+1}-G_{i} u^{n}} & \text { if } G_{i} u^{n} \neq G_{i} u^{n+1} \\ 0 & \text { if } G_{i} u^{n}=G_{i} u^{n+1} \geq 0 \\ \frac{1}{2 \epsilon}\left(G u^{n+1}+G u^{n}\right) & \text { if } G_{i} u^{n}=G_{i} u^{n+1}<0\end{cases}
$$

Setting $\mathcal{E}_{\text {pen }}^{n}:=\mathcal{E}^{n}+\frac{1}{2 \epsilon}\left(\left(G u^{n}\right)^{-}\right)^{2}$, the energy balance reads

$$
\mathcal{E}_{\text {pen }}^{n+1}-\mathcal{E}_{\text {pen }}^{n}={ }^{\mathrm{T}} F^{n+\frac{1}{2}}\left(u^{n+1}-u^{n}\right)
$$

\subsection{Explicit schemes}

We can also envisage an explicit scheme for Problem 5.1. 

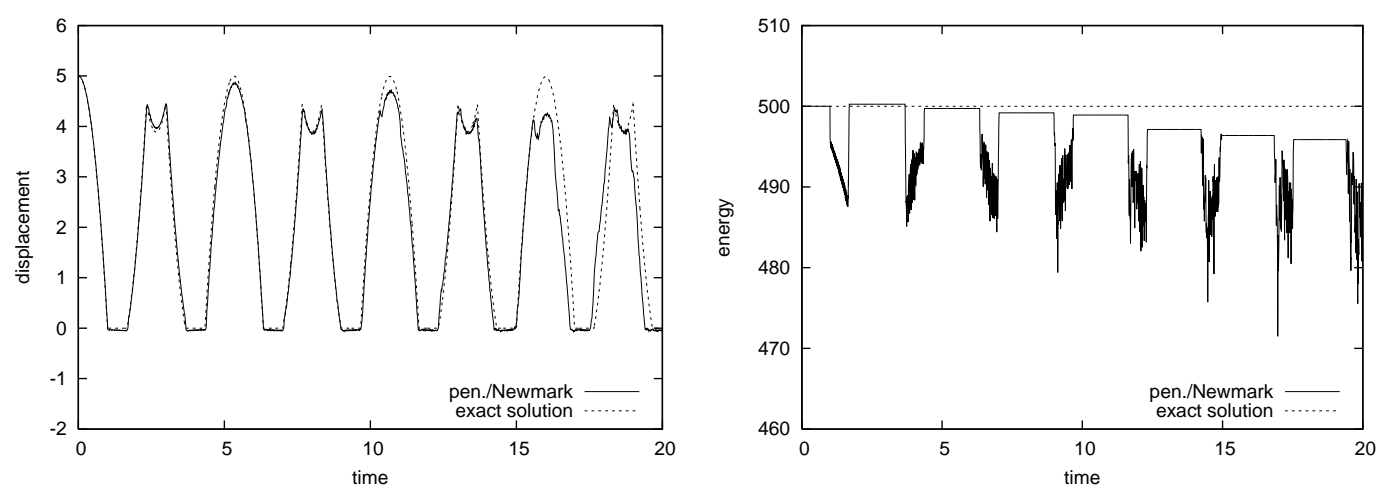

Figure 19. Bounces of an elastic bar. Displacement $u_{0}^{n}$ (left) and energy $\mathcal{E}^{n}-{ }^{\mathrm{T}} F u^{n}$ (right). Discretization 5.1 with $\alpha=0, \beta=1 / 4$ and $\gamma=1 / 2, \epsilon=10^{-4} . \Delta x=0.1, \Delta t=0.005, \nu_{c}=1.5$.

Discretization 5.3 (Central differences) Seek $u^{n+1} \in \mathbb{R}^{N_{d}}$ such that

$$
M\left(\frac{u^{n+1}-2 u^{n}+u^{n-1}}{\Delta t^{2}}\right)+K u^{n}=F^{n}+{ }^{\mathrm{T}} G R_{\epsilon}\left(G u^{n}\right) .
$$

The penalty term stiffens the system of ODEs, which limits the stability domain of the schemes. This is not surprising since the penalty contact condition tends to the exact contact condition when $1 / \epsilon$ tends to infinity and an explicit exact enforcement of the contact condition is not possible. A more detailed discussion on the choice of the penalty parameter can be found in [3].

\section{DISCRETIZATIONS WITH CONTACT CONDITION IN VELOCITY}

In this part, standard finite elements in space are combined with an approximation of the contact condition involving the velocity.

Problem 6.1. Seek a displacement $u:[0, T] \rightarrow \mathbb{R}^{N_{d}}$ and a contact pressure $r:[0, T] \rightarrow \mathbb{R}^{N_{c}}$ such that, for almost every $t \in[0, T]$,

$$
\begin{aligned}
& M \ddot{u}(t)+K u(t)=f(t)+{ }^{\mathrm{T}} G r(t) \\
& G \dot{u}(t) \geq 0, \quad r(t) \geq 0, \quad{ }^{\mathrm{T}} r(t) G \dot{u}(t)=0
\end{aligned}
$$

with the initial conditions $u(0)=u^{0}$ and $\dot{u}(0)=v^{0}$.

With this contact condition in velocity, the semi-discrete problem is much simpler than (32)(33). Problem 6.1 is still a system of differential equations under unilateral constraints, but the constraint involves now the velocity instead of the displacement. The general theory developed in $[9,11]$ applies to Problem 6.1. The solution $u$ is unique. Furthermore, $u$ is continuous and $\dot{u}$ is differentiable in time almost everywhere, so that the equations have a sense at almost every time. The mathematical analysis of this problem can be found in [9]. The time discretization 
has been extensively studied in [11].

Unfortunately, the contact condition in velocity is not equivalent to the Signorini condition. This approximation is valid only on a short time interval after an impact. The strategy adopted is the following: if a node satisfies the non-interpenetration condition, then at the next iteration no constraint is enforced on this node; if a node breaks the non-interpenetration condition, then at the next iteration the contact condition in velocity will be applied to this node. This approach allows for slight interpenetration. At each time step, we define the matrix $G^{n}$ whose rows $G_{i}^{n}$ are

$$
G_{i}^{n}= \begin{cases}(0 \ldots 0) & \text { if } G_{i} u^{n} \geq 0 \\ G_{i} & \text { if } G_{i} u^{n}<0\end{cases}
$$

This approach based on a contact condition in velocity has also been widely used in rigid-body dynamics with impacts.

\subsection{Implicit schemes}

A midpoint scheme with a contact condition velocity has been proposed in [22].

Discretization 6.1 (Midpoint [22]) Seek $u^{n+1}, \dot{u}^{n+1} \in \mathbb{R}^{N_{d}}$, and $r^{n+1} \in \mathbb{R}^{N_{c}}$ such that

$$
\begin{aligned}
& M \ddot{u}^{n+\frac{1}{2}}+K u^{n+\frac{1}{2}}=F^{n+\frac{1}{2}}+{ }^{\mathrm{T}} G^{n} r^{n+\frac{1}{2}}, \\
& G^{n} \dot{u}^{n+\frac{1}{2}} \geq 0, \quad r^{n+\frac{1}{2}} \geq 0, \quad{ }^{\mathrm{T}} r^{n+\frac{1}{2}} G^{n} \dot{u}^{n+\frac{1}{2}}=0, \\
& u^{n+1}=u^{n}+\Delta t \dot{u}^{n+\frac{1}{2}}, \\
& \dot{u}^{n+1}=\dot{u}^{n}+\Delta t \ddot{u}^{n+\frac{1}{2}} .
\end{aligned}
$$

An interesting feature of this scheme is to be energy-conserving,

$$
\mathcal{E}^{n+1}-\mathcal{E}^{n}={ }^{\mathrm{T}} F^{n+\frac{1}{2}}\left(u^{n+1}-u^{n}\right) .
$$

The contact pressure does not perturb the structure despite its oscillations (Figure 20). Owing to the energy conservation, the long-time behavior is quite satisfactory (Figure 21).

\subsection{Semi-explicit schemes}

In [3], an explicit scheme based on the contact condition in velocity has been proposed.

Discretization 6.2 (Central differences [3]) Seek $u^{n+1} \in \mathbb{R}^{N_{d}}$ and $r^{n+1} \in \mathbb{R}^{N_{c}}$ such that

$$
\begin{aligned}
& M\left(\frac{u^{n+1}-2 u^{n}+u^{n-1}}{\Delta t^{2}}\right)+K u^{n}=F^{n}+{ }^{\mathrm{T}} G^{n} r^{n+1}, \\
& G^{n}\left(u^{n+1}-u^{n}\right) \geq 0, \quad r^{n+1} \geq 0, \quad{ }^{\mathrm{T}} r^{n+1} G^{n}\left(u^{n+1}-u^{n}\right)=0 .
\end{aligned}
$$

Numerical simulations suggest that the stability condition of the central difference scheme is not tightened by the contact condition. The numerical results are similar to those obtained with Discretization 4.7 (Figures 22 and 23). 

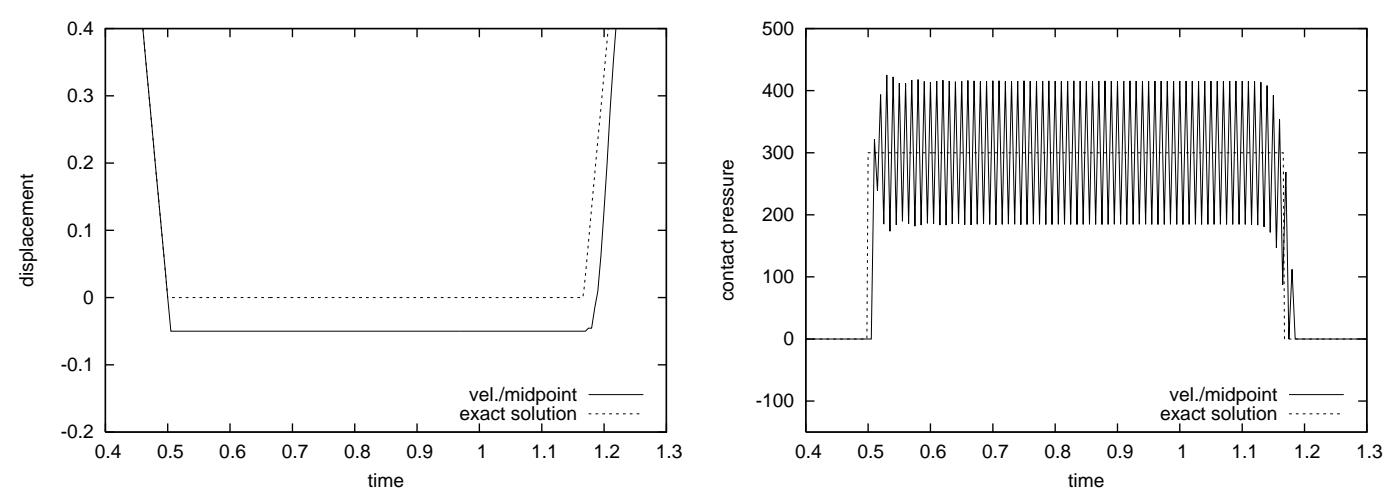

Figure 20. Impact of an elastic bar. Displacement $u_{0}^{n}$ (left) and stress (right). Discretization 6.1 with $\alpha=0, \beta=1 / 4$ and $\gamma=1 / 2 . \Delta x=0.1, \Delta t=0.005, \nu_{c}=1.5$.
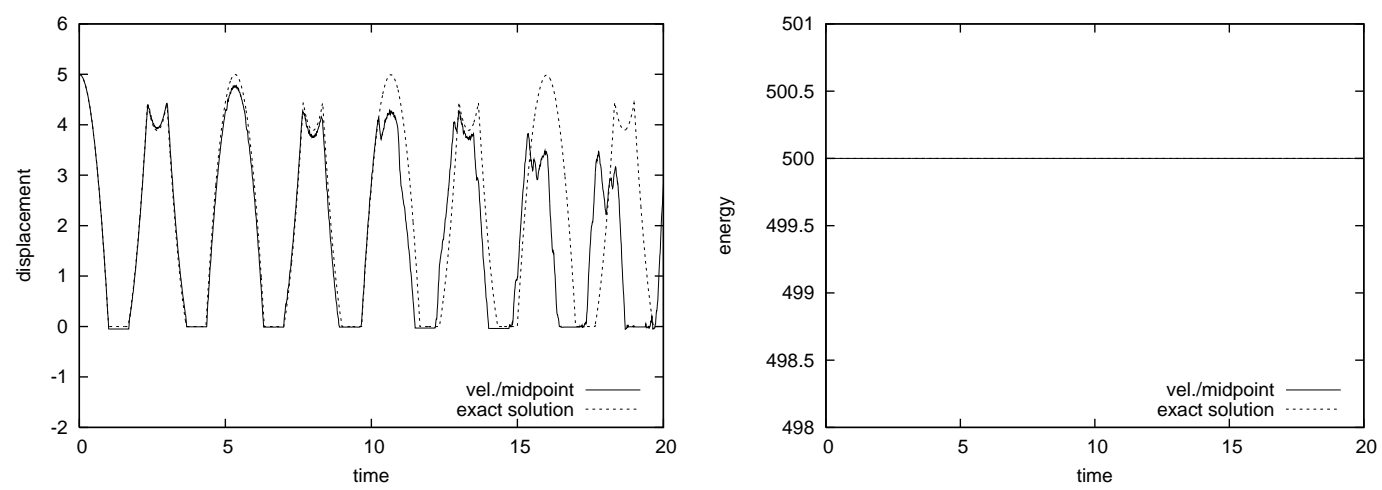

Figure 21. Bounces of an elastic bar. Displacement $u_{0}^{n}$ (left) and energy $\mathcal{E}^{n}-{ }^{\mathrm{T}} F u^{n}$ (right). Discretization 6.1 with $\alpha=0, \beta=1 / 4$ and $\gamma=1 / 2 . \Delta x=0.1, \Delta t=0.005, \nu_{c}=1.5$.

\section{DISCRETIZATIONS WITH MODIFIED MASS}

In the previous three parts, we have considered various ways of enforcing the contact condition. Here we describe methods based on a modification of the mass matrix. Such methods are thus compatible with any enforcement of the contact condition. For brevity, we restrict ourselves to an exact enforcement of the contact condition. In the modified mass matrix, the entries associated with the normal displacements at the contact boundary are set to zero. The motivation for this modification is very simple: if the mass is removed, the inertial forces and the oscillations are eliminated. This approach has been introduced in [18].

Set $N_{d}^{*}:=N_{d}-N_{c}$. For the sake of simplicity, suppose that the degrees of freedom associated with normal displacements at the contact boundary are numbered from $N_{d}^{*}+1$ to $N_{d}$. The 

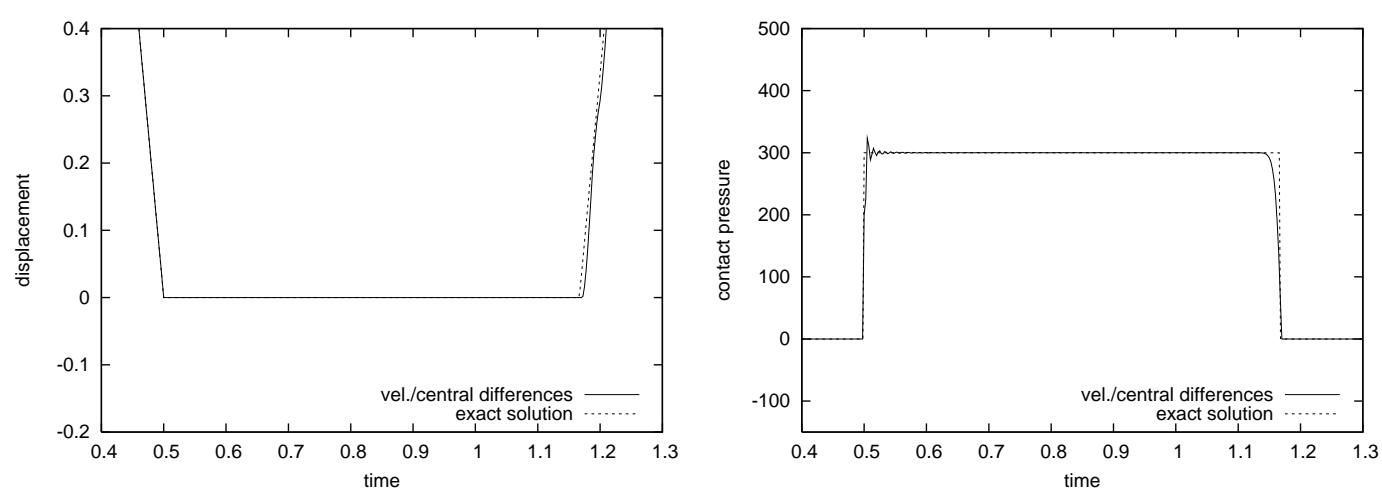

Figure 22. Impact of an elastic bar. Displacement $u_{0}^{n}$ (left) and contact pressure $r^{n}$ (right). Discretization 6.2 (lumped mass matrix). $\Delta x=0.1, \Delta t=0.0025, \nu_{c}=0.75$.
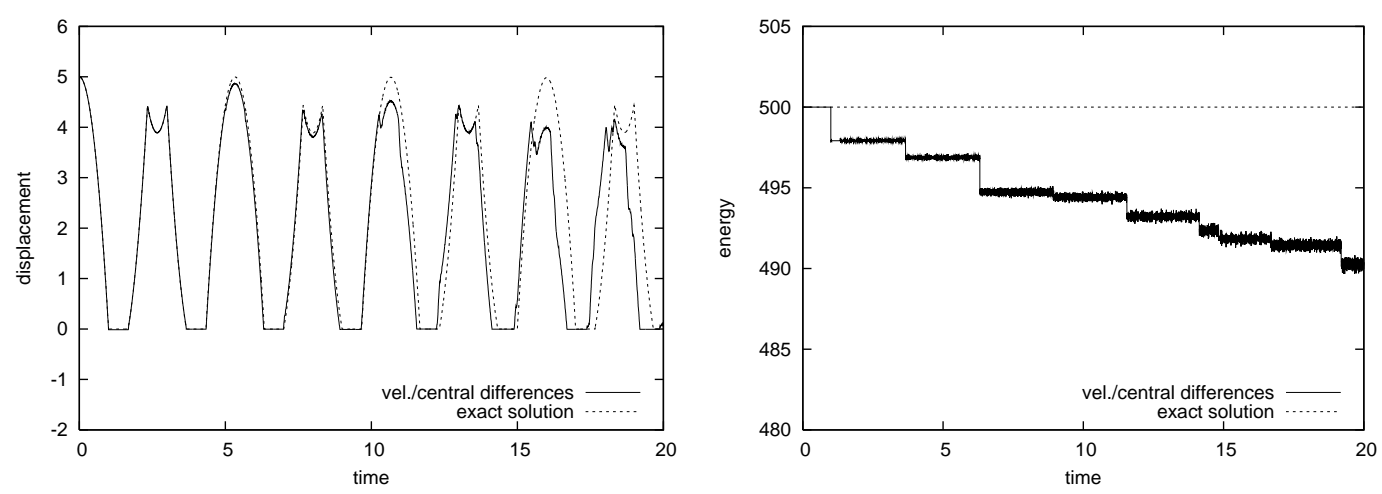

Figure 23. Bounces of an elastic bar. Displacement $u_{0}^{n}$ (left) and energy $\mathcal{E}^{n}-{ }^{\mathrm{T}} F u^{n}$ (right). Discretization 6.2 (lumped mass matrix). $\Delta x=0.1, \Delta t=0.0025, \nu_{c}=0.75$.

modified mass matrix is defined as

$$
M^{*}=\left(\begin{array}{ll}
M_{* *} & 0 \\
0 & 0
\end{array}\right) .
$$

Many choices are possible to build the block $M_{* *}$. In [13, 18], the authors devise various methods to preserve some features of the standard mass matrix (the total mass, the center of gravity and the moments of inertia). We can also simply take the corresponding block in the standard mass matrix (and this is what will be done in our numerical simulations below). The modified problem reads

$$
\begin{aligned}
& M^{*} \ddot{u}(t)+K u(t)=F(t)+{ }^{\mathrm{T}} G r(t), \\
& G u(t) \geq 0, \quad r(t) \geq 0, \quad{ }^{\mathrm{T}} r(t) G u(t)=0 .
\end{aligned}
$$


If we set $u(t)=\left(\begin{array}{c}u_{*}(t) \\ u_{c}(t)\end{array}\right), K=\left(\begin{array}{ll}K_{* *} & K_{* c} \\ K_{c *} & K_{c c}\end{array}\right), F(t)=\left(\begin{array}{c}F_{*}(t) \\ F_{c}(t)\end{array}\right)$, and $G=\left(G_{*} G_{c}\right)$, then equations (97) and (98) can be recast as

$$
\begin{aligned}
& M_{* *} \ddot{u}_{*}(t)+K_{* *} u_{*}(t)+K_{* c} u_{c}(t)=F_{*}(t), \\
& K_{c *} u_{*}(t)+K_{c c} u_{c}(t)=F_{c}(t)+{ }^{\mathrm{T}} G_{c} r(t), \\
& G_{c} u_{c}(t) \geq 0, \quad r(t) \geq 0, \quad{ }^{\mathrm{T}} r(t) G_{c} u_{c}(t)=0 .
\end{aligned}
$$

For a given $t$ and a given $u_{*}(t)$, there exists one and only one $u_{c}(t)$ satisfying (100) and (101). Denote by $Q:[0, T] \times \mathbb{R}^{N_{d}^{*}} \rightarrow \mathbb{R}^{N_{c}}$ the non-linear application such that $u_{c}(t)=Q\left(t, u_{*}(t)\right)$.

Problem 7.1. Seek a displacement $u:[0, T] \rightarrow \mathbb{R}^{N_{d}}$ such that, for all $t \in[0, T]$,

$$
\begin{aligned}
& M_{* *} \ddot{u}_{*}(t)+K_{* *} u_{*}(t)+K_{* c} Q\left(t, u_{*}(t)\right)=F_{*}(t), \\
& u_{c}(t)=Q\left(t, u_{*}(t)\right),
\end{aligned}
$$

with the initial conditions $u(0)=u^{0}$ and $\dot{u}(0)=v^{0}$.

The operator $Q(t, \cdot)$ is Lipschitz continuous at each time $t$, so that equation (102) is a Lipschitz system of ODEs. Therefore, it has a unique solution $u_{*}$, twice differentiable in time. Owing to (103), $u_{c}$ is differentiable in time almost everywhere. The detailed mathematical analysis of the semi-discrete modified mass formulation can be found in [18, 8]. A result of convergence of the semi-discrete solutions to a continuous solution is proven for viscoelastic materials in [8].

Remark 7.1. In contrast to the semi-discrete problem with standard mass matrix, the semidiscrete problem with modified mass matrix does not require an impact law and conserves the energy.

\subsection{Implicit schemes}

Let us discretize Problem 7.1 with a HHT-Newmark scheme.

Discretization 7.1 (HHT-Newmark [18]) Seek $u^{n+1} \in \mathbb{R}^{N_{d}}$, $\dot{u}_{*}^{n+1} \in \mathbb{R}^{N_{d}}$ and $\ddot{u}_{*}^{n+1} \in$ $\mathbb{R}^{N_{d}}$ such that

$$
\begin{aligned}
& M_{* *} \ddot{u}_{*}^{n+1}+K_{* *} u_{*}^{n+1+\alpha}+K_{* c} Q\left(t^{n+1+\alpha}, u_{*}^{n+1+\alpha}\right)=F_{*}^{n+1+\alpha}, \\
& u_{c}^{n+1+\alpha}=Q\left(t^{n+1+\alpha}, u_{*}^{n+1+\alpha}\right), \\
& u_{*}^{n+1}=u_{*}^{n}+\Delta t \dot{u}_{*}^{n}+\frac{\Delta t^{2}}{2} \ddot{u}_{*}^{n+2 \beta}, \\
& \dot{u}_{*}^{n+1}=\dot{u}_{*}^{n}+\Delta t \ddot{u}_{*}^{n+\gamma} .
\end{aligned}
$$

The equations can be recast as a linear complementarity problem,

$$
\begin{aligned}
& M^{*} \ddot{u}^{n+1}+K u^{n+1+\alpha}=F^{n+1+\alpha}+{ }^{\mathrm{T}} G r^{n+1}, \\
& G u^{n+1} \geq 0, \quad r^{n+1} \geq 0, \quad{ }^{\mathrm{T}} r^{n+1} G u^{n+1}=0, \\
& u^{n+1}=u^{n}+\Delta t \dot{u}^{n}+\frac{\Delta t^{2}}{2} \ddot{u}^{n+2 \beta}, \\
& \dot{u}^{n+1}=\dot{u}^{n}+\Delta t \ddot{u}^{n+\gamma} .
\end{aligned}
$$


In spite of the modification of the mass matrix, the problem is well posed. In practice, we use this set of equations to compute the solution. As expected, the large oscillations have disappeared during the contact phase (Figure 24). The energy conservation is also very satisfactory (Figure 25), since there holds

$$
\mathcal{E}_{*}^{n+1}-\mathcal{E}_{*}^{n}={ }^{\mathrm{T}}\left(\frac{r^{n+1}+r^{n}}{2}\right)\left(G u^{n+1}-G u^{n}\right)+{ }^{\mathrm{T}}\left(\frac{F^{n+1}+F^{n}}{2}\right)\left(u^{n+1}-u^{n}\right),
$$

where $\mathcal{E}_{*}^{n}$ has the same expression than $\mathcal{E}^{n}$, except that $M$ is replaced by $M^{*}$.
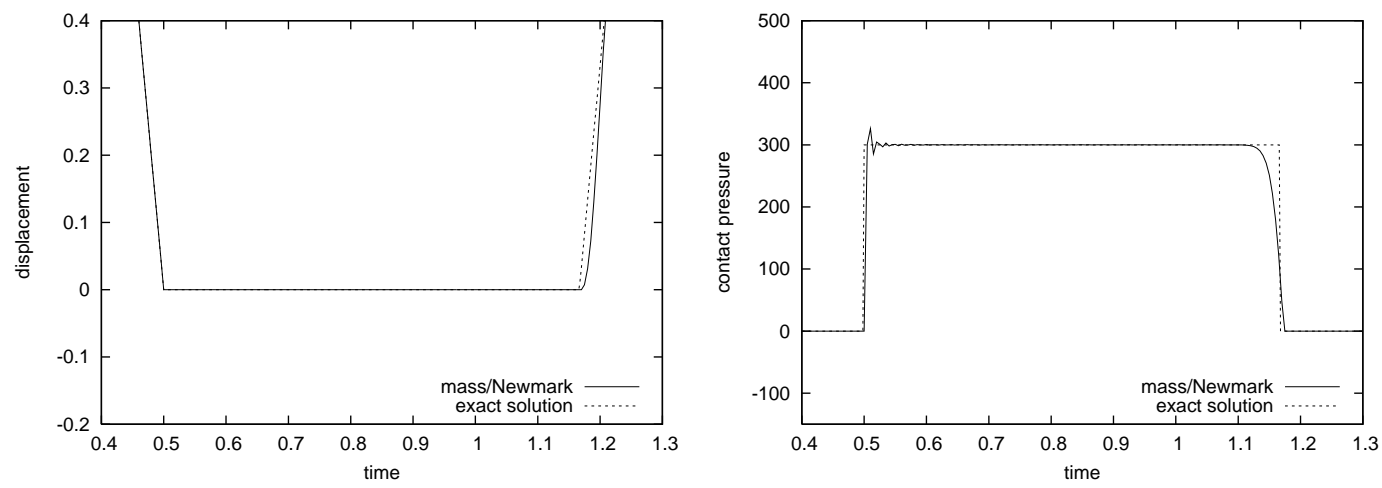

Figure 24. Impact of an elastic bar. Displacement $u_{0}^{n}$ and contact pressure $r^{n}$. Discretization 7.1 with $\alpha=0, \beta=1 / 4$ and $\gamma=1 / 2 . \Delta x=0.1, \Delta t=0.005, \nu_{c}=1.5$.
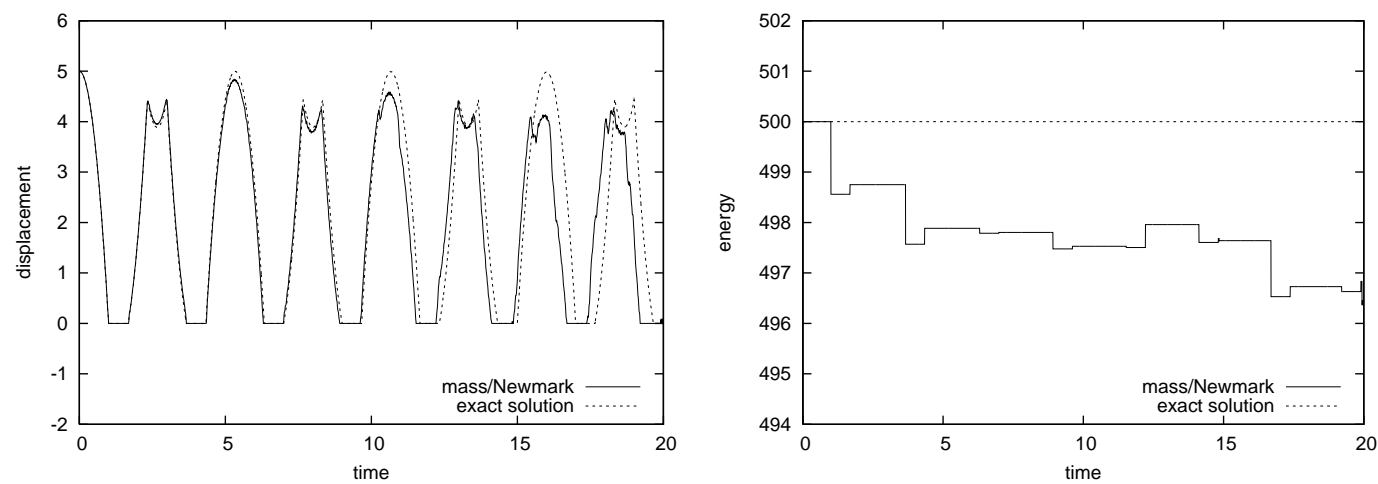

Figure 25. Bounces of an elastic bar. Displacement $u_{0}^{n}$ (left) and energy $\mathcal{E}_{*}^{n}-{ }^{\mathrm{T}} F u^{n}$ (right). Discretization 7.1 with $\alpha=0, \beta=1 / 4$ and $\gamma=1 / 2 . \Delta x=0.1, \Delta t=0.005, \nu_{c}=1.5$.

\subsection{Semi-explicit schemes}

The modified mass method was introduced with implicit schemes. But we can also discretize Problem 7.1 with an explicit scheme, such as the central difference scheme. This yields a new 
semi-explicit scheme.
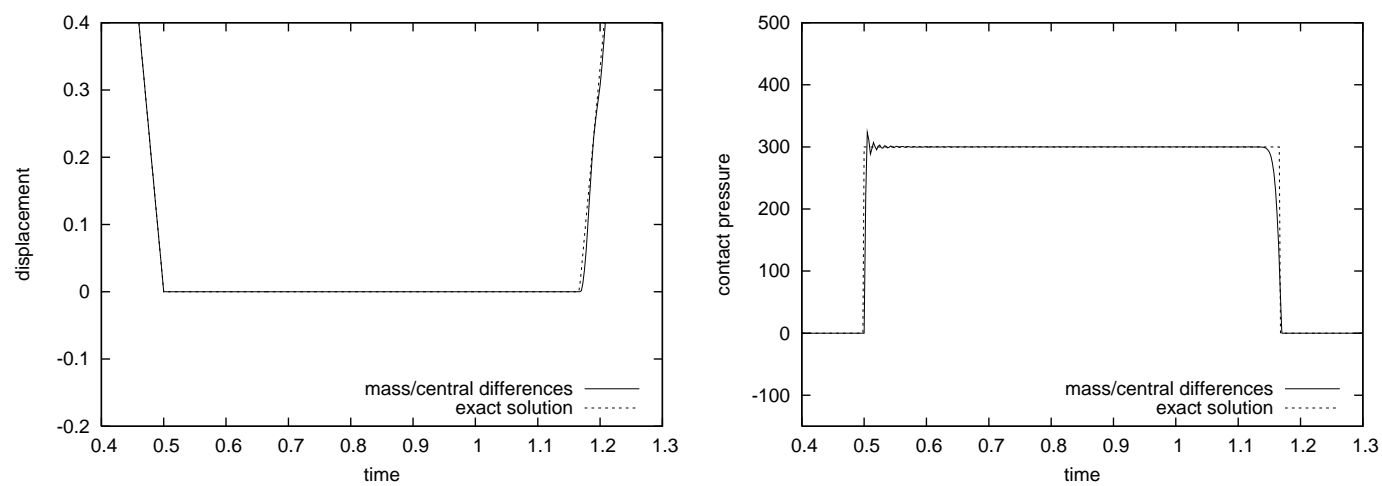

Figure 26. Bounces of an elastic bar. Displacement $u_{0}^{n}$ (left) and contact pressure $r^{n}$ (right). Discretization 7.2 (lumped mass matrix). $\Delta x=0.1, \Delta t=0.0025, \nu_{c}=0.75$.
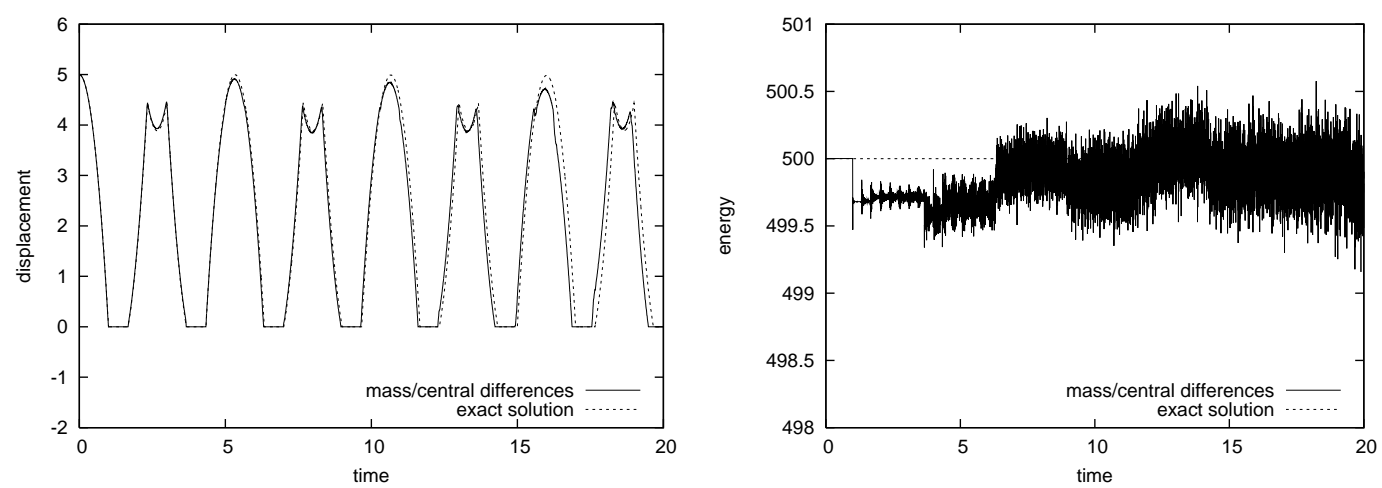

Figure 27. Bounces of an elastic bar. Displacement $u_{0}^{n}$ (left) and energy $\mathcal{E}_{*}^{n}-{ }^{\mathrm{T}} F u^{n}$ (right). Discretization 7.2 (lumped mass matrix). $\Delta x=0.1, \Delta t=0.0025, \nu_{c}=0.75$.

Discretization 7.2 (Central differences) Seek $u^{n+1} \in \mathbb{R}^{N_{d}}$ such that

$$
\begin{aligned}
& M_{* *}\left(\frac{u_{*}^{n+1}-2 u_{*}^{n}+u_{*}^{n-1}}{\Delta t^{2}}\right)+K_{* *} u_{*}^{n}+K_{* c} Q\left(t^{n}, u_{*}^{n}\right)=F_{*}^{n}, \\
& u_{c}^{n+1}=Q\left(t^{n+1}, u_{*}^{n+1}\right) .
\end{aligned}
$$

In practice, the equations are solved the following way. 1. Seek $u_{*}^{n+1} \in \mathbb{R}^{N_{d}}$ such that

$$
M_{* *}\left(\frac{u_{*}^{n+1}-2 u_{*}^{n}+u_{*}^{n-1}}{\Delta t^{2}}\right)+K_{* *} u_{*}^{n}+K_{* c} u_{c}^{n}=F_{*}^{n} .
$$

Copyright (c) 2000 John Wiley \& Sons, Ltd.

Int. J. Numer. Meth. Engng 2000; 00:1-6 Prepared using nmeauth.cls 
2. Seek $u_{c}^{n+1} \in \mathbb{R}^{N_{d}^{*}}$ and $r^{n+1} \in \mathbb{R}^{N_{c}}$ such that

$$
\begin{aligned}
& K_{c *} u_{*}^{n+1}+K_{c c} u_{c}^{n+1}=F_{c}^{n+1}+{ }^{\mathrm{T}} G_{c} r^{n+1}, \\
& G_{c} u_{c}^{n+1} \geq 0, \quad r^{n+1} \geq 0, \quad{ }^{\mathrm{T}} r G_{c} u_{c}^{n+1}=0 .
\end{aligned}
$$

The first step is explicit, provided the mass matrix $M_{* *}$ is lumped. The second step is a constrained problem which concerns only the variable $u_{c}$. Discretization 7.2 satisfies the system

$$
\begin{aligned}
& M^{*}\left(\frac{u^{n+1}-2 u^{n}+u^{n-1}}{\Delta t^{2}}\right)+K u^{n}=F^{n}+{ }^{\mathrm{T}} G r^{n}, \\
& G u^{n} \geq 0, \quad r^{n} \geq 0, \quad{ }^{\mathrm{T}} r^{n} G u^{n}=0,
\end{aligned}
$$

and the energy balance,

$$
\mathcal{E}_{0, \frac{1}{2} *}^{n+1}-\mathcal{E}_{0, \frac{1}{2} *}^{n}={ }^{\mathrm{T}}\left(\frac{r^{n+1}+r^{n}}{2}\right)\left(G u^{n+1}-G u^{n}\right)+{ }^{\mathrm{T}}\left(\frac{F^{n+1}+F^{n}}{2}\right)\left(u^{n+1}-u^{n}\right),
$$

where $\mathcal{E}_{0, \frac{1}{2} *}^{n}$ has the same expression than $\mathcal{E}_{0, \frac{1}{2}}^{n}$, except that $M$ is replaced by $M^{*}$. We observe numerically that the stability condition on the time step is the same as in the linear case. Compared with Discretizations 4.7 and 6.2, the semi-explicit modified mass method shows a better energy conservation and a better long-time behavior (Figure 27).

\section{APPENDIX}

When approximating a solution presenting a shock or a sharp wave front with finite elements in space and a time-stepping scheme, spurious oscillations are observed. This is the wellknown Gibbs phenomenon which is due to the poor approximation of eigenmodes associated with the high frequencies. In the elastodynamic Signorini problem, we must deal with shock waves after the impacts. If we plot the stress distribution computed with the modified mass method and a Newmark scheme, we see this phenomenon (Figure 28). Moreover, the magnitude of the oscillations does not decrease with the time step. For instance, as observed in [12], with small time steps, the oscillations appears in front of the shock instead of behind. These oscillations can be eliminated by using dissipative schemes, such as the HHT or Chung-Hulbert schemes (or, which is equivalent, by filtering). For instance, the damping obtained with a HHT scheme is presented in Figure 29. It may seem a little bit contradictory to discard the use of dissipative schemes for the contact oscillations and to reintroduce them for the Gibbs phenomenon. Actually, the oscillations on the stress caused by the Gibbs phenomenon are far smaller than those caused by the contact condition (compare Figure 6 with Figure 28). The former are proportional to the discontinuity jump, the latter are proportional to $\frac{m_{i} v_{i}}{\Delta t}$, where $v_{i}$ is the velocity before the impact and $m_{i}$ is the mass associated with the impacting node. Consequently, the amount of dissipation needed is much smaller.

\section{REFERENCES}

1. F. Armero and E. Petocz. Formulation and analysis of conserving algorithms for frictionless dynamic contact/impact problems. Comput. Methods Appl. Mech. Engrg., 158(3-4):269 - 300, 1998. 

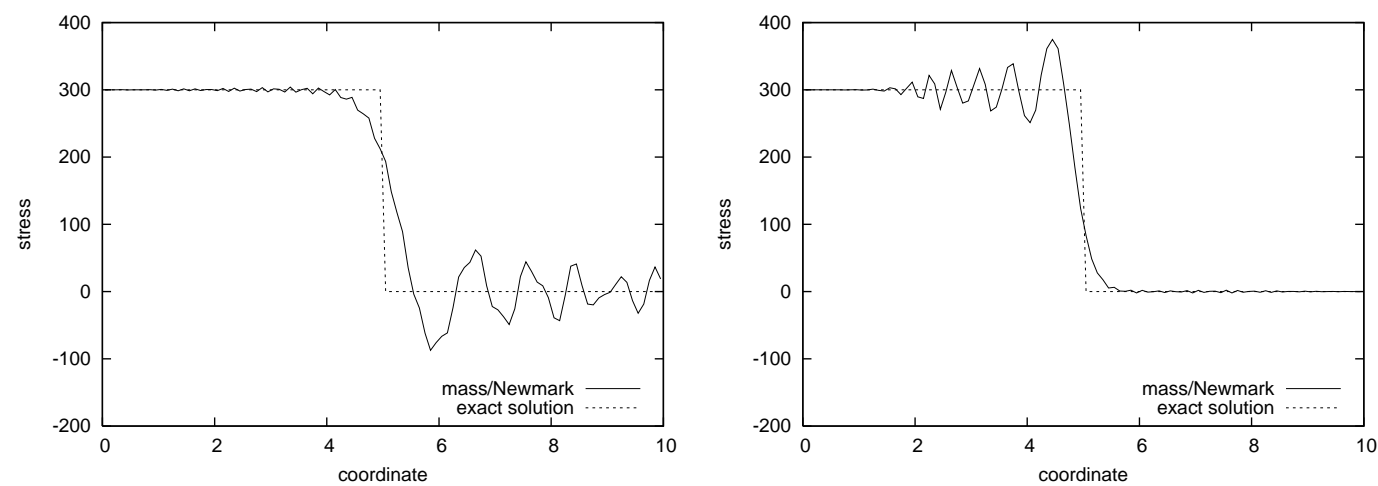

Figure 28. Impact of an elastic bar. Stress in the bar at time $\mathrm{t}=1.0$. Discretization $7.1 . \Delta x=0.1$. $\Delta t=0.005, \nu_{c}=1.5$ (left). $\Delta t=0.00125, \nu_{c}=0.375$ (right).
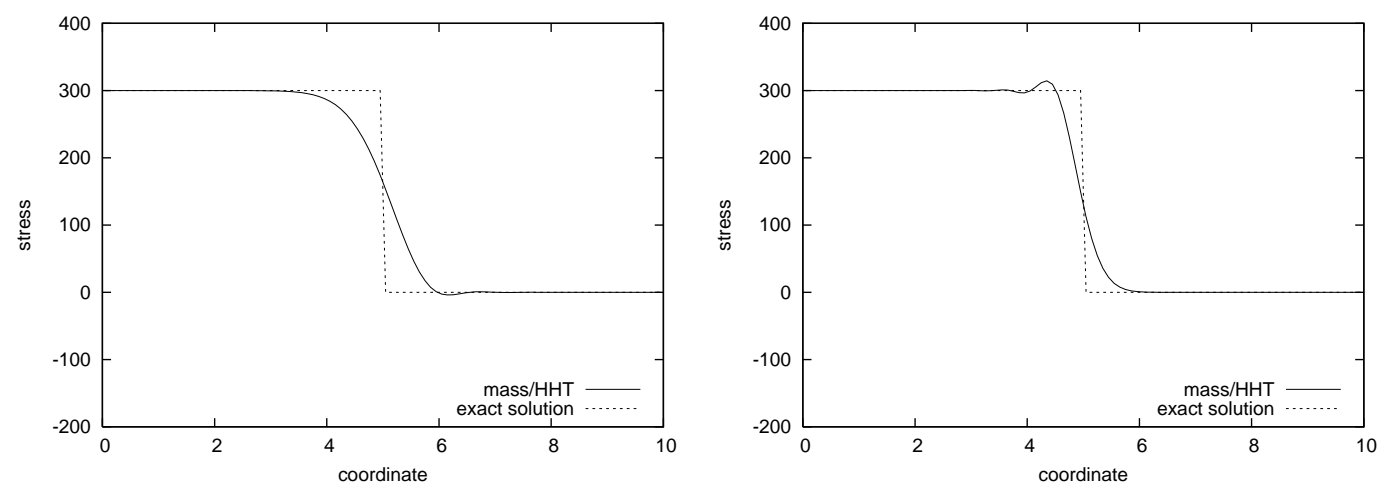

Figure 29. Impact of an elastic bar. Stress in the bar at time $t=1.0$. Discretization 7.1 with $\alpha=-0.06$, $\beta=1 / 4(1-\alpha)^{2}, \gamma=1 / 2-\alpha . \Delta x=0.1 . \Delta x=0.1 . \Delta t=0.005, \nu_{c}=1.5$ (left). $\Delta t=0.00125$, $\nu_{c}=0.375$ (right).

2. Y. Ayyad, M. Barboteu, and J. Fernandez. A frictionless viscoelastodynamic contact problem with energy consistent properties: Numerical analysis and computational aspects. Comput. Methods Appl. Mech. Engrg., 198(5-8):669 - 679, 2009.

3. T. Belytschko and M. Neal. Contact-impact by the pinball algorithm with penalty and lagrangian methods. Int. J. Numer. Methods Engrg., 31(3):547-572, 1991.

4. B. Brogliato. Nonsmooth impact mechanics. Springer-Verlag London Ltd., London, 1996.

5. N. Carpenter, R. Taylor, and M. Katona. Lagrange constraints for transcient finite element surface contact. Int. J. Numer. Meth. Engng, 32:103-128, 1991.

6. R. Courant and D. Hilbert. Methods of mathematical physics. Vol. I. Interscience Publishers, Inc., New York, N.Y., 1953.

7. P. Deufhard, R. Krause, and S. Ertel. A contact-stabilized Newmark method for dynamical contact problems. Int. J. Numer. Methods Engrg., 73(9):1274-1290, 2008.

8. D. Doyen and A. Ern. Convergence of a space semi-discrete modified mass method for the dynamic signorini problem. Commun. Math. Sci. (to appear).

9. G. Duvaut and J.-L. Lions. Inequalities in mechanics and physics. Springer-Verlag, Berlin, 1976.

10. C. Eck, J. Jarusek, and M. Krbec. Unilateral contact problems. Chapman \& Hall/CRC, Boca Raton, 
FL, 2005. Variational methods and existence theorems.

11. R. Glowinski, J.-L. Lions, and R. Trémolières. Numerical analysis of variational inequalities. NorthHolland Publishing Co., Amsterdam, 1981.

12. E. Grosu and I. Harari. Stability of semidiscrete formulations for elastodynamics at small time steps. Finite Elements in Analysis and Design, 43(6-7):533 - 542, 2007.

13. C. Hager, S. Hüeber, and B. I. Wohlmuth. A stable energy-conserving approach for frictional contact problems based on quadrature formulas. Int. J. Numer. Methods Engrg., 73(2):205-225, 2008.

14. P. Hauret and P. Le Tallec. Energy-controlling time integration methods for nonlinear elastodynamics and low-velocity impact. Comput. Methods Appl. Mech. Engrg., 195(37-40):4890-4916, 2006.

15. T. J. R. Hughes. The finite element method. Prentice Hall Inc., Englewood Cliffs, NJ, 1987.

16. G. M. Hulbert and J. Chung. Explicit time integration algorithms for structural dynamics with optimal numerical dissipation. Comput. Methods Appl. Mech. Engrg., 137(2):175 - 188, 1996.

17. C. Kane, E. A. Repetto, M. Ortiz, and J. E. Marsden. Finite element analysis of nonsmooth contact. Comput. Methods Appl. Mech. Engrg., 180(1-2):1-26, 1999.

18. H. B. Khenous, P. Laborde, and Y. Renard. Mass redistribution method for finite element contact problems in elastodynamics. European Journal of Mechanics - A/Solids, 27(5):918 - 932, 2008.

19. N. Kikuchi and J. T. Oden. Contact problems in elasticity: a study of variational inequalities and finite element methods. SIAM, Philadelphia, PA, 1988.

20. S. Krenk. Energy conservation in Newmark based time integration algorithms. Comput. Methods Appl. Mech. Engrg., 195(44-47):6110-6124, 2006.

21. T. A. Laursen. Computational contact and impact mechanics. Springer-Verlag, Berlin, 2002.

22. T. A. Laursen and V. Chawla. Design of energy conserving algorithms for frictionless dynamic contact problems. Int. J. Numer. Methods Engrg., 40(5):863-886, 1997.

23. T. A. Laursen and G. R. Love. Improved implicit integrators for transient impact problems-geometric admissibility within the conserving framework. Int. J. Numer. Methods Engrg., 53(2):245-274, 2002.

24. G. Lebeau and M. Schatzman. A wave problem in a half-space with a unilateral constraint at the boundary. J. Differential Equations, 53(3):309-361, 1984

25. L. Paoli and M. Schatzman. A numerical scheme for impact problems. I. The one-dimensional case. SIAM J. Numer. Anal., 40(2):702-733), 2002.

26. L. Paoli and M. Schatzman. A numerical scheme for impact problems. II. The multidimensional case. SIAM J. Numer. Anal., 40(2):734-768, 2002.

27. M. Schatzman and M. Bercovier. Numerical approximation of a wave equation with unilateral constraints. Math. Comp., 53(187):55-79, 1989.

28. D. E. Stewart. Rigid-body dynamics with friction and impact. SIAM Rev., 42(1):3-39, 2000.

29. R. Taylor and P. Papadopoulos. On a finite element method for dynamic contact/impact problems. Int. J. Numer. Meth. Engng, 36:2123-2140, 1993.

30. D. Vola, E. Pratt, M. Jean, and M. Raous. Consistent time discretization for dynamical contact problems and complementarity techniques. Revue Européenne des Eéments Finis, 7(1-3):149-162, 1998.

31. P. Wriggers. Computational Contact Mechanics. John Wiley \& Sons Ltd., 2002. 